\title{
Kavalierstour: Kulturkontakte und Fremdheitserfahrungen
}

\author{
David Winkler-Ebner \\ Kerngebiet: Neuzeit \\ eingereicht bei: ao. Univ.-Prof. Dr. Heinz Noflatscher \\ eingereicht im: SoSe 2017 \\ Rubrik: SE-Arbeit
}

\begin{abstract}
Grand Tour: Cultural Contact and Encounters with the Other

The following seminar paper is about the Grand Tour of European noblemen in the early modern period. It will examine which cultural aspects were primarily perceived by the travellers and which evidence of feelings of "strangeness" or "otherness" appears in their travel reports. As will be shown, the travellers paid a lot of attention to architecture and historiography, but there is hardly any evidence of feelings of otherness regarding these topics, whereas language, clothing and cuisine are hardly ever mentioned but if they are, it always happens in a context in which the travellers experienced alterity.
\end{abstract}

\section{Einleitung}

Etwa zu Beginn des 16. Jahrhunderts nahm europaweit die geographische Mobilität von Studenten, aber auch Professoren, sehr stark zu. Unter den Herumziehenden befanden sich auch immer mehr Adelige, die damit auf die zunehmenden kulturellen und intellektuellen Anforderungen an ihren Stand reagierten. In der vorliegenden Arbeit, die einen Beitrag zur Mobilitätsgeschichte europäischer Studenten in der Frühen Neuzeit leisten will, soll es vor allem um die Reisetätigkeit Adeliger gehen und dabei um ein Phänomen, das in der Forschung Grand Tour oder Kavalierstour - in weiterer Folge soll ausschließlich letzterer der beiden Begriffe verwendet werden - genannt wird. Den Begriff definiert Katrin Keller als „eine von spezifisch adligen Normen geprägte Bildungsreise", die zwar nicht ausschließlich vom Geburtsadel unternommen, 
jedoch vorrangig „der Integration der Reisenden in die höfisch geprägte Adelskultur des Absolutismus" diente. ${ }^{1}$

Wie jede Reise - damals wie heute - führte die Kavalierstour zu interkulturellen Kontakten und dieser Aspekt soll in der Arbeit besonders beleuchtet werden. Konkret soll es um die Frage gehen, welche kulturellen Aspekte von den Reisenden besonders wahrgenommen wurden und welche Hinweise auf Fremdheitserfahrungen sich aus den schriftlich fixierten Beobachtungen erschließen lassen.

Dabei sollen mehrere Thesen bzw. Behauptungen anderer Historiker_innen überprüft werden. Zum einen die eigene These, dass in der Frühen Neuzeit, die ja oft auch als „konfessionelles Zeitalter" bezeichnet wird, ${ }^{2}$ die religiösen Aspekte eine zentrale Rolle spielten und damit auch starke Fremdheitsgefühle bei Andersartigkeit verbunden waren.

Weiters soll die These von Hilde Ridder-Symoens, frühneuzeitliche Reiseberichte würden „die Entwicklung nationaler Stereotypen und Vorurteile" fördern, ${ }^{3}$ überprüft werden.

Außerdem sollen einige von Wolfgang Treues ${ }^{4}$ Behauptungen in Bezug auf Fremdheitserfahrungen in der Frühen Neuzeit einer Untersuchung unterzogen werden. So waren es laut Treue etwa Frauen und deren Lebensumstände, die auf Reisen sehr genau beobachtet wurden. Die Konfrontation mit fremden Religionen wiederum bildete ihm zufolge so etwas wie eine "Grenze der Offenheit". Andersartigkeit hatte, so Treue weiter, in Bezug auf Nahrungsmittel und Tischsitten einen besonders hohen Stellenwert in der Fremdheitserfahrung frühneuzeitlicher Reisender.

Die Vorgehensweise dabei ist folgende: In der Arbeit soll exemplarisch ein Reisebericht, der im Zuge einer adeligen Kavalierstour der Frühen Neuzeit verfasst wurde, dahingehend untersucht werden, ob die hier genannten Thesen und Behauptungen (auch) auf diesen Bericht zutreffen. Nach einer äußeren sowie inneren Kritik der Quelle erfolgt eine qualitative und quantitative Inhaltsanalyse des Reiseberichts. Dabei kommt Philipp Mayerings Modell ${ }^{5}$ einer strukturierenden Inhaltsanalyse zur Anwendung und zwar in Form der Unterkategorie des Modells, der inhaltlichen Strukturierung.

1 Katrin Keller, Zwischen Zeremoniell und "desbauche“. Die adlige Kavalierstour um 1700, in: Wolfgang Schmale/ Reinhard Stauber (Hrsg.), Menschen und Grenzen in der Frühen Neuzeit (Innovation. Bibliothek zur Neueren und Neuesten Geschichte 2), Berlin 1998, S. 259-282, hier S. 260.

2 Michael Maurer, ,Nationalcharakter' in der frühen Neuzeit. Ein mentalitätsgeschichtlicher Versuch, in: Helmut Kuzmics/Reinhard Blomert/Annette Treibel (Hrsg.), Transformationen des Wir-Gefühls. Studien zum nationalen Habitus, Frankfurt a. M. 1993, S. 45-81, hier S. 46.

$3 \quad$ Hilde de Ridder-Symoens, Die Kavalierstour im 16. und 17. Jahrhundert, in: Peter J. Brenner (Hrsg.), Der Reisebericht. Die Entwicklung einer Gattung in der deutschen Literatur, Frankfurt a. M. 1989, S. 197-223, hier S. 219.

$4 \quad$ Wolfgang Treue, Abenteuer und Anerkennung. Reisende und Gereiste im Spätmittelalter und Früher Neuzeit, Paderborn 2014.

$5 \quad$ Philipp Mayering, Qualitative Inhaltsanalyse, Weinheim-Basel 2012. 
Dem Analyseteil, in dem ein Reisebericht Johann Georg Keyßlers ${ }^{6}$ untersucht wird, geht ein längerer Theorieteil voraus, in dem zunächst das Phänomen der Kavalierstour umrissen wird. Wichtige Arbeiten hierzu stammen etwa von Hilde de Ridder-Symoens ("Die Kavalierstour im 16. und 17. Jahrhundert" ${ }^{\prime 7}$ ), Antje Stannek ("Telemachs Brüder" sowie Werner Paravicini („Vom Erkenntniswert der Adelsreise ${ }^{\prime \prime}$ ).

Anschließend werden einige allgemeine Bemerkungen zu frühneuzeitlicher Reiseliteratur und der in der Frühen Neuzeit beginnenden Methodisierung des Reisens gemacht. Intensiv mit diesem Thema auseinandergesetzt hat sich Justin Stagl, etwa in seinem Werk „Eine Geschichte der Neugier"10. Auch Peter J. Brenners Sammelband „Der Reisebericht in der deutschen Literatur"111 sei hier erwähnt.

Zuletzt soll näher auf das Phänomen der "Fremde“ in der Frühen Neuzeit eingegangen werden. Besonders hervorzuheben ist Wolfgang Treues "Abenteuer und Anerkennung "12 sowie der Sammelband von Wolfgang Schmale und Reinhard Stauber "Menschen und Grenzen in der Frühen Neuzeit"

\section{Kavalierstour}

\subsection{Vorläufer und Entwicklung der Kavalierstour}

„Der Umgang mit Menschen verhilft dem menschlichen Urteil zu herrlicher Klarheit. [...] All die verschiedenen Temperamente, Glaubensrichtungen, Urteile, Meinungen, Gesetze und Bräuche lehren uns, die eigenen kritisch zu beurteilen und einzusehen, daß [sic!] unsere Urteilskraft unvollkommen, von Natur aus schwach ist."14

So schrieb im 16. Jahrhundert der Philosoph Michel de Montaigne in einem seiner Essays und lieferte damit der Historikerin Hilde de Ridder-Symoens einen eindrücklichen Beweis für ihre These, schon die Humanisten seien vom Wert und Nutzen des Reisens überzeugt gewesen. ${ }^{15}$ Zum einen, so Ridder-Symoens, glaubten diese durch die Be-

6 Johann Georg Keyßler, Neueste Reisen durch Deutschland, Böhmen, Ungarn, die Schweiz, Italien und Lothringen [...], Hannover 1751, S. XIX-LXXVII, [https://books.google.at/books/about/Johann_Georg_Keysslers_Neueste_ Reisen_du.html?id=W9AWAAAAQAAJ\&redir_esc=y], eingesehen 24.8.2017.

$7 \quad$ Ridder-Symoens, Kavalierstour.

8 Antje Stannek, Telemachs Brüder. Die höfische Bildungsreise des 17. Jahrhunderts (Geschichte und Geschlechter 33), Frankfurt a. M. 2001

9 Werner Paravicini, Vom Erkenntniswert der Adelsreise: Einleitung, in: Rainer Babel/Werner Paravicini (Hrsg.), Grand Tour. Adliges Reisen und Europäische Kultur vom 14. bis zum 18. Jahrhundert. Akten der internationalen Kolloquien in der Villa Vigoni 1999 und im Deutschen Historischen Institut Paris 2000 (Beihefte der Francia 60), Ostfildern 2005, S. 11-20.

10 Justin Stagl, Eine Geschichte der Neugier. Die Kunst des Reisens 1550-1800, Wien-Köln-Weimar 2002.

11 Peter J. Brenner, Die Erfahrung der Fremde. Zur Entwicklung einer Wahrnehmungsform in der Geschichte des Reiseberichts, in: Peter J. Brenner (Hrsg.), Der Reisebericht in der deutschen Literatur, Frankfurt a. M. 1989, S. 14-49.

12 Treue, Abenteuer

13 Wolfgang Schmale/Reinhard Stauber (Hrsg.), Menschen und Grenzen in der Frühen Neuzeit (Innovation. Bibliothek zur Neueren und Neuesten Geschichte 2), Berlin 1998.

14 Michel de Montaigne, Les Essais de Michel de Montaigne I, hrsg. v. Pierre Villey, Paris 1922, S. 202-203., zit. in Hilde de Ridder-Symoens, Mobilität, in: Walter Rüegg (Hrsg.), Geschichte der Universität in Europa II: Von der Reformation bis zur Französischen Revolution 1500-1800, München 1996, S. 335-359, hier S. 335. 
gegnung mit fremden Völkern ihr Urteilsvermögen zu schärfen und ihren Bildungshorizont zu erweitern. Diese Ansicht vertrat offenbar auch Montaigne, wie aus obigem Zitat hervorgeht. Zum anderen nutzten die Humanisten die Reise ins Ausland für den Fremdsprachenerwerb. ${ }^{16}$ Da Aufgeschlossenheit, Universalität und Kosmopolitismus zu den „wesentlichen Elementen der humanistischen Weltanschauung"17 gehörten, führten diese beiden Aspekte - die Erweiterung des persönlichen Horizonts und die Möglichkeit, die eigenen Kenntnisse fremder Sprachen zu vertiefen - laut Ridder-Symoens zu einem starken Anstieg der geographischen Mobilität von Studenten, aber auch Professoren an der Wende zum 16. Jahrhundert. Die Studienreise ins Ausland wurde mehr und mehr zum "krönende[n] Abschluß humanistischer Bildung”, wie die Historikerin es nennt. ${ }^{18}$ Damit setzte eine neue Blüte der peregrinatio academica, der Bildungs- und Gelehrtenreise, die bereits im Mittelalter existiert hatte, ein. ${ }^{19}$

Hauptziel dieser Frühform frühneuzeitlicher Bildungsreisen war Italien - schließlich lagen hier nicht nur die "Quellen der antiken Zivilisation“20, die die Humanisten aufzuspüren trachteten, auch der Humanismus selbst hatte hier seinen Ursprung genommen. ${ }^{21}$ Besucht wurden vor allem die Städte Bologna, Padua, Siena, Pavia und Pisa, an deren Universitäten man Medizin oder Jurisprudenz in Kombination mit den Werken antiker Autoren studierte; und dies durchaus mit heißem Bemühen. Laut Ridder-Symoens weisen die durchschnittliche Dauer des Studienaufenthalts und der hohe Anteil an Promotionen auf die Ernsthaftigkeit hin, mit der ausländische Studenten ihr Studium am iter italicum betrieben.22

Im Zuge der Konfessionalisierung änderte der Grundsatz des 1555 geschlossenen Augsburger Religionsfriedens, cuius regio, eius religio, die Situation entscheidend. Die Landesherren versuchten ihren Untertanen den Besuch ausländischer Universitäten zu verwehren, da sie darin eine Gefahr für deren konfessionelle und politische Loyalität sahen. Stattdessen strebten die Herrscher nach der raschen Errichtung eigener Universitäten, in denen Beamte und Geistliche nach ihren Vorstellungen ausgebildet werden sollten. Drei Arten von Hochschulen entstanden dabei: protestantische (etwa Wittenberg, Heidelberg, Genf und Straßburg), katholische (u. a. Paris, Wien, Löwen, Köln, Dole, Graz, Würzburg und die Universitäten der iberischen Halbinsel) und bewusst tolerante Universitäten, an denen auch Studenten anderer Konfessionen zugelassen wurden, etwa Padua, Siena, Orléans, Leiden oder Montpellier. ${ }^{23}$

Auf der anderen Seite jedoch bedeutete dies eine starke Zuwanderungsbewegung der Studenten an die Universitäten ihrer jeweiligen Konfession - ein Hinweis darauf, dass es den damals schwach ausgebauten Staatsapparaten nicht gelang, die Verbote

Ridder-Symoens, Mobilität, S. 335.

Ridder-Symoens, Kavalierstour, S. 198.

Ridder-Symoens, Mobilität, S. 335.

Ebd., S. 197.

Ridder-Symoens, Kavalierstour, S. 197.

Ebd.

Ridder-Symoens, Mobilität, S. 336.

Ebd., S. 337-339. 
wirkungsvoll umzusetzen. ${ }^{24}$ Diese konfessionsabhängige Wanderung führte etwa dazu, dass Italien nicht mehr länger das Zielland erster Wahl für ausländische Studenten war - im Gegenteil: Da protestantische Studenten nun vor allem ins Römisch-Deutsche Reich zogen, verloren Universitäten wie Bologna, Ferrara, Perugia und Rom, die allesamt im Kirchenstaat lagen, den Großteil ihrer ausländischen Studenten. ${ }^{25}$

Die hier schemenhaft aufgezeigte Entwicklung der studentischen Migration in Europa seit dem Beginn der Frühen Neuzeit (mit Wurzeln im Mittelalter) ist einer der Stränge, die zur Entstehung der adeligen Kavalierstour führten. Ein weiterer verläuft über die Geschichte adeligen Reisens seit dem europäischen Spätmittelalter, die Werner Paravicini in vier Abschnitte unterteilt: Heidenfahrt, Pilgerfahrt, Ritterfahrt und Kavalierstour. ${ }^{26}$ Auch Ronald Asch erkennt in der Kavalierstour eine Fortsetzung der spätmittelalterlichen Adelsreise, die seiner Ansicht nach dem Sammeln militärischer Erfahrungen und damit als eine Art Kreuzzugsersatz diente. Als Beispiel nennt Asch den auch von Paravicini erwähnten Kampf gegen „Heiden“ in Litauen. ${ }^{27}$ Diese Ansicht steht allerdings im Widerspruch zu Paravicinis Ausführungen, in denen festgehalten wird, dass die Pilgerfahrt ohne kriegerische Elemente auskam. ${ }^{28}$ Es ist somit davon auszugehen, dass Asch einen ganz konkreten Typ im Kopf hat, wenn er den Begriff spätmittelalterliche Adelsreise verwendet. Unter dem oben erwähnten Begriff Ritterfahrt versteht Paravicini indes eine Form des Reisens, die die Elemente der Heidenfahrt und der Pilgerfahrt verband und bei der als neues Element der "systematische Besuch der Höfe" hinzutrat. Außerdem steigt dabei die Neugierde der Reisenden vom Laster zur Tugend auf. Wichtig ist nun, „wunderbare Dinge“ zu sehen - und noch viel wichtiger: sagen zu können, wunderbare Dinge gesehen zu haben. ${ }^{29}$ Katrin Keller verweist ebenso auf eine Traditionslinie der Adelsreise seit dem Mittelalter. ${ }^{30}$

Paravicini erwähnt an anderer Stelle eine weitere Tradition, in der die Kavalierstour der Frühen Neuzeit stand. Es war dies die Praxis, junge männliche Adelige ab einem bestimmten Zeitpunkt zur weiteren Ausbildung an einen Verwandten oder Nachbarn, zum Teil auch an einen nahen oder sehr angesehenen Fürstenhof, zu geben. ${ }^{31}$

Schließlich nennt Keller noch die Bestrebungen des Frühabsolutismus, den Adel zu domestizieren, ihn einerseits zu entmachten und andererseits durch die Zentralisierung von Herrschaft zur physischen Präsenz am Hof zu zwingen, einen weiteren Wegbereiter der Kavalierstour, zu dessen wesentlichen Elementen der Besuch fremder Höfe gehörte. $^{32}$

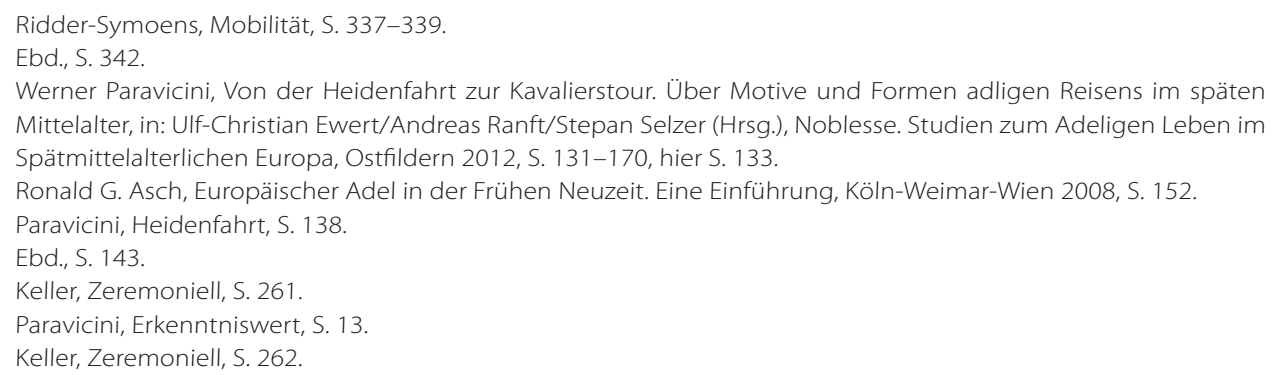


Was waren nun die konkreten Gründe, die die Entstehung der frühneuzeitlichen Kavalierstour veranlassten? Als wesentlich zu nennen ist hier eine Veränderung des adeligen Bildungsideals - denn Gelehrsamkeit galt dem Adel lange Zeit als "unstandesgemäß"33. Paravicini spricht in dem Zusammenhang von „alter Verachtung des [...] Schriftwerks" ${ }^{\prime 34}$. Dies führte dazu, dass laut Asch der Universitätsbesuch für die meisten Adeligen bis zur Mitte des 16. Jahrhunderts eher etwas Ungewöhnliches darstellte - wenn sie nicht eine kirchliche Laufbahn anstrebten. ${ }^{35}$ Laut Asch fand also im 16. Jahrhundert ein Umdenken in Bezug auf adelige Bildung statt, Paravicini erkennt ein solches schon etwas früher, in der zweiten Hälfte des 15. Jahrhunderts. ${ }^{36}$ Bis dahin galt der Adel zeitgenössischen Kritikern als „oberflächlich kultiviert”. Tatsächlich beschränkte sich das adelige Bildungsinteresse lange Zeit auf Pferde, Hunde, die Jagd, das Kriegshandwerk, Genealogie und die Landwirtschaft. ${ }^{37}$

Das diesbezügliche Umdenken sieht Keller im Zusammenhang mit dem Prozess der „Umgruppierung und Neuorientierung der Führungsschichten", bei dem es in erster Linie um soziale Distinktion gegenüber dem Bürgertum ging. Dies war im Zuge des "Auf- und Ausbau[s] moderner Staatlichkeit und differenzierter Verwaltung"38 - Keller nennt als diesbezüglichen Zeitpunkt ebenso wie Asch die Mitte des 16. Jahrhunderts - notwendig geworden, da nun gelehrte Bürgerliche zu ernstzunehmenden Konkurrenten des Adels im Kampf um die höchsten Ämter wurden und der Adel in dieser Hinsicht immer mehr an Boden zu verlieren drohte. ${ }^{39}$ Die weltliche Elite erkannte, so Paravicini, dass sie um ein Studium nicht mehr umhin kam, „um der Konkurrenz der gelehrten Räte mit ihrer lateinischen Wohlredenheit und juristischen Spitzfindigkeit standhalten zu können." ${ }^{\prime 40}$ In einem ersten Schritt, so Keller, ging es um die Nachholung akademischer Bildungsdefizite des Adels gegenüber dem Bürgertum. ${ }^{41}$ Paravicini folgert: "Damit wurde die Universität in den Kanon der Adelsreise aufgenommen.“42 Doch die Änderungen waren weitreichender: Um seine Vorrangstellung gegenüber niederen sozialen Schichten zu bewahren, gestaltete der Adel seine Bildung in solcher Weise um, dass akademisch-gelehrte Bildung mit dem Ideal des "Hofmannes" verbunden wurde. Letzterer zeichnete sich aus durch "geschliffene Umgangsformen", die "Fähigkeit zu galant-gebildeter Konversation", "Sprach- und Weltkenntnis" sowie die "Ausbildung in verschiedenen ,adligen Exercitien'”. Dieses Hofmanns-Ideal wurde nun zur Voraussetzung für den Aufstieg in hohe Ämter erklärt. ${ }^{43}$ Der Wandel zu diesem Ideal hin war eine wesentliche Voraussetzung für die Entstehung der Kavalierstour, wie Keller, Paravicini, Asch und Ridder-Symoens schreiben. Das lag zum einen daran,

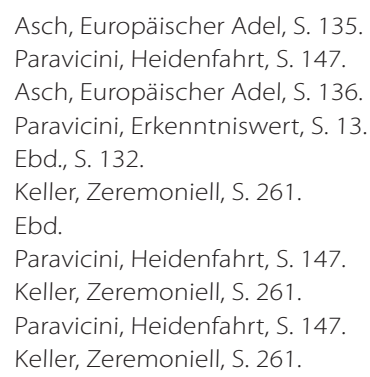


dass besagtes Bildungsideal nur durch den Besuch von Höfen erreicht werden konnte, vorzugsweise jener, die "den kulturellen Stand der Zeit" verkörperten. ${ }^{44}$ Zum anderen erforderte die nun als ebenso wichtig erachtete gelehrte Bildung, die den gestiegenen Ansprüchen im Staatsdienst genügen sollte, den Besuch von Universitäten in Ländern, wo "moderne Kenntnisse in Mathematik, Kriegs- und Befestigungstechnik, Staatskunst, Verwaltungslehre, Diplomatie, aber auch in der schönen Literatur und den bildenden Künsten" erworben werden konnten. ${ }^{45}$

Antje Stannek hingegen ist in Bezug auf die Gründe für die Zunahme der Besuche ausländischer Universitäten durch Adlige skeptischer. Sie spricht diesbezüglich von einer kontroversen Diskussion über die Ursachen und hält lediglich fest, dass der Anstieg der adeligen Studenten im Ausland ein europaweites Phänomen war, das im 16. Jahrhundert begann - auch Stannek datiert also später als Paravicini - und sich im 17. Jahrhundert fortsetzte. ${ }^{46}$

Die erste Hochphase der Kavalierstour erkennt Keller am Beginn des 17. Jahrhunderts, was sie an der steigenden Zahl diesbezüglicher Reiseberichte sowie einer „Blüte apodemischer Literatur", die als Leitfaden für die Reise dienten, festmacht. Eine Zäsur kam mit dem Dreißigjährigen Krieg, der die Praxis der Kavalierstour bei Adeligen im Alten Reich fast zur Gänze zum Erliegen brachte. Erst ab den 1650er-Jahren nahm die Zahl der Reisen wieder zu und eine zweite Hochphase setzte ein, die laut Keller bis ins zweite Drittel des 18. Jahrhunderts dauerte. ${ }^{47}$

Bezüglich des Auslaufens der Praxis der Kavalierstour scheiden sich die Geister der Historiker_innen. Während Keller den Niedergang der Kavalierstour mit Anfang des 18. Jahrhunderts beginnen lässt und das späteste Ende zur Zeit des Siebenjährigen Krieges (1756-1763) ansetzt, ${ }^{48}$ behauptet Paravicini, es bestehe ein Konsens in der Forschung, dass die Kavalierstour ihr Ende mit der Französischen Revolution ab dem Jahr 1789 und den darauffolgenden Napoleonischen Kriegen nahm. ${ }^{49}$ Ridder-Symoens datiert den Niedergang der Kavalierstour bereits ins 17. Jahrhundert. ${ }^{50}$ Als Gründe hierfür nennt Asch die zunehmenden Maßnahmen der Landesherren, die durch Verbote das Auslandsstudium ihrer Untertanen (wie schon in der Zeit nach der Reformation, s. o.) zu unterbinden versuchten. Verbunden war damit ein Ausbau der heimischen Bildungseinrichtungen, in denen die Erziehung zu loyalen „Dienern des Staates” im Vordergrund stand, was zudem die Notwendigkeit eines Auslandsstudiums verringerte. ${ }^{51}$ Ridder-Symoens ergänzt als Grund für den Rückgang der Kavalierstour die europäischen Konflikte des 17. Jahrhunderts, vor allem die Kriege Ludwigs XIV.52 Ihr zufolge kam die studentische Wanderung in der Zeit der Aufklärung zwar nicht zum Erliegen

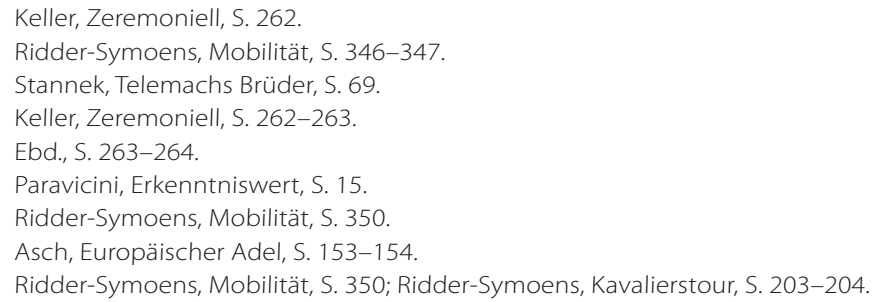


- im Gegenteil: Die Aufklärung brachte ihrer Meinung nach durchaus eine Belebung des universitären Lebens und einen gewissen Anstoß für die Bildungsmigration - allerdings ging diese nun kaum mehr über die Landesgrenzen hinaus..$^{53}$

\subsection{Reiserouten}

Neben den oben erwähnten Universitäten und Höfen wurden auch andere Einrichtungen besucht, und zwar die in Europa ausgehend von Italien neu entstandenen Adelsakademien, die dem neuen adeligen Bildungsideal entsprachen, indem sie die gelehrte Bildung verbanden mit Studien, die dem Ideal des Hofmannes entsprachen etwa Reiten, Fechten, Tanzen und Sprachen. ${ }^{54}$

Ursprünglich waren diese Einrichtungen auf Bestreben der jeweiligen Landesherren entstanden, um die einheimischen Eliten im eigenen Land zu halten. ${ }^{55}$ In Italien entstanden auf diese Weise, so Ridder-Symoens, die Collegi dei Nobili, in Frankreich die académies (laut Stannek auch: collèges) ${ }^{56}$, im Alten Reich nannten man sie Ritterakademien, in Spanien Colegios Mayores, in den Vereinigten Niederlanden gymnasia illustria und in England wiederum colleges. ${ }^{57}$ Ironischerweise entwickelten sich aber gerade diese Akademien und Gymnasien, die im Übrigen keine akademischen Grade vergeben konnten, zu äußerst beliebten Zielen auf der Kavalierstour, was daran lag - glaubt man Ridder-Symoens - dass sich dort der hochnäsige Jungadel wohler fühlte als an den Universitäten, auch wenn diese sich in eine ähnliche Richtung entwickelten. ${ }^{58}$

Wohin führten nun die Reisen der Kavaliere? Dies hing verständlicherweise auch von deren eigener Herkunft ab. Darum soll zunächst festgehalten werden, aus welchen Ländern diese stammten. Grundsätzlich, so schreibt Ridder-Symoens, handelte es sich bei den Kavalieren um Vertreter der Eliten aller europäischen Länder. Die Mehrheit stellten aber eindeutig Engländer und Bewohner des Reichs dar. Aber auch das holländische Prinzipat sowie der polnische, ungarische und tschechische Adel begaben sich häufig auf die Reise. Ridder-Symoens Formel dazu lautet: „Protestanten mehr als Katholiken, Angelsachsen, Deutschsprachige, Slawen stärker als Franzosen, Italiener und Spanier."59

Das wichtigste Ziel war wie schon bei der humanistischen peregrinatio academica Italien, ${ }^{60}$ wo wiederum stets Rom das Hauptziel der Reise darstellte. ${ }^{61}$ Die Gründe hierfür waren der gute Ruf der dortigen Adelsakademien sowie der direkte Zugang zu den Kunstschätzen der Antike und der Renaissance. ${ }^{62}$ Mit der zunehmenden Orientierung der höfischen Kultur an Frankreich trat dieses als Zielland der Kavalierstour immer

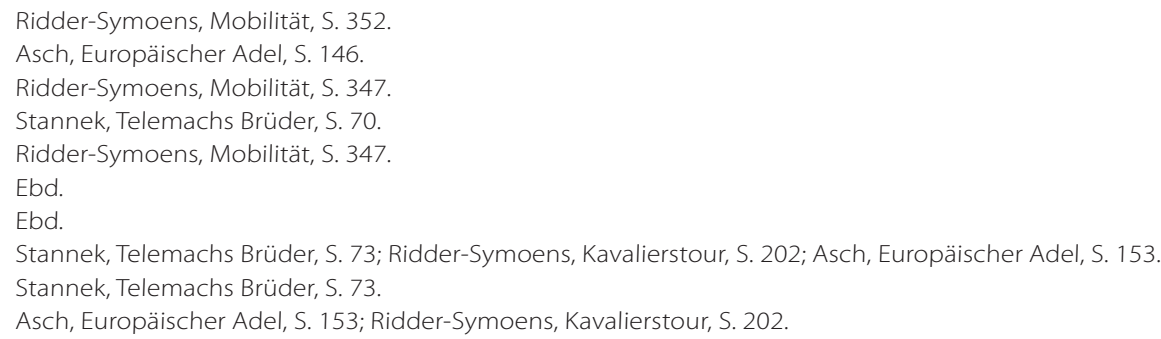


stärker in Konkurrenz zu Italien, ${ }^{63}$ was laut Asch und Ridder-Symoens nicht zuletzt auch an den dort ansässigen traditionsreichen Universitäten und guten Akademien lag. ${ }^{64}$ Keller schreibt dazu, dass ab der zweiten Hälfte des 17. Jahrhunderts Frankreich eindeutig zum Hauptziel der Kavalierstour aufgestiegen war. ${ }^{65}$ Auch England und die Vereinigten Niederlande waren häufige Ziele, da sich dort die Reisenden mit der neuartigen Regierungsform des Parlamentarismus vertraut machen konnten. ${ }^{66}$ Das Alte Reich stand nur bei wenigen Kavalieren auf dem Plan - starken Anteil daran hatten, wie schon erwähnt, die Verwüstungen durch den Dreißigjährigen Krieg. Wenn doch jemand kam, dann stammte er zumeist aus Mittel- oder Osteuropa. ${ }^{67}$ Auch Spanien, das als gefährlich und teuer galt, wurde selten besucht, ${ }^{68}$ ebenso wie die Länder Skandinaviens. ${ }^{69}$

Die Ziele der Kavalierstour waren also stark eingeschränkt und eine räumliche Einschränkung erkennt Keller auch innerhalb der einzelnen Länder: Die Kavaliere steuerten überwiegend große Städte, Residenzen, Universitäten und Akademien an und waren bemüht, die Wege dazwischen rasch zu überwinden. Der Erfahrungsraum der Kavaliere, so Keller, war folglich ein überwiegend "städtischer".70

\subsection{Funktionen der Kavalierstour}

Dass es sich bei der Kavalierstour um eine Bildungsreise handelte, die zudem der soziaIen Distinktion diente, wurde bereits deutlich. Im Hinblick darauf sei noch erwähnt, dass es grundsätzlich keine ständisch begründete Beschränkung des Zugangs zum neuen adeligen Bildungsideal gab. Warum Bürgerliche dennoch kaum dieses Ideal erreichten, lag zum einen an dem damit verbundenen großen Aufwand, zum anderen daran, dass die Erziehung zum „Hofmann", die neben gelehrter Bildung den zweiten zentralen Bestandteil adeliger Bildung darstellte, nur durch den Besuch von Höfen erfolgen konnte. Zu diesen hatten - vom Adel abgesehen - nur eine "schmale bürgerlich-patrizische Elite" Zugang, wie Keller schreibt.71

Die Kavalierstour erfüllte darüber hinaus aber noch andere Zwecke. Als zentral nennt Stannek hier die "Kontaktaufnahme mit der höfischen Welt".72 Diese bedeutet nicht nur den Erwerb höfischer Umgangsformen, ${ }^{73} \mathrm{~d}$. h. die Erziehung zum bereits angesprochenen Ideal des Hofmannes, sondern auch, den eigenen Platz in der Standeshierarchie kennenzulernen sowie die Fähigkeit zu erwerben, die "Platzierungsmechanismen" innerhalb der höfischen Gesellschaft zu durchschauen. ${ }^{74}$ Zudem war darunter der Auf-

63 Stannek, Telemachs Brüder, S. 77; Asch, Europäischer Adel, S. 153; Ridder-Symoens, Kavalierstour, S. 202.

64 Asch, Europäischer Adel, S. 153; Ridder-Symoens, Kavalierstour, S. 202.

65 Keller, Zeremoniell, S. 268.

66 Ridder-Symoens, Kavalierstour, S. 202.

67 Ridder-Symoens, Mobilität, S. 349.

68 Ridder-Symoens, Kavalierstour, S. 202.

69 Keller, Zeremoniell, S. 268.

70 Ebd., S. 269.

71 Ebd., S. 265

72 Stannek, Telemachs Brüder, S. 14

73 Keller, Zeremoniell, S. 264; Ridder-Symoens, Kavalierstour, S. 200

74 Stannek, Telemachs Brüder, S. 13 
bau eines Beziehungsnetzwerkes zu verstehen, das später in diplomatischer oder militärischer Hinsicht relevant sein konnte. ${ }^{75}$ Darüber hinaus bot die Reise die Möglichkeit zur "Aneignung prestigeträchtiger Sachgüter" wie überhaupt die Mobilität den jungen Adeligen für den Statuserwerb diente. ${ }^{76}$

Stannek spricht von der Kavalierstour weiters als einem von vielen Initiationsritualen in der Frühen Neuzeit. Weitere Beispiele wären die Firmung oder die Schwertleite, das zeremonielle Umgürten mit der Waffe, das meist im 21. Lebensjahr, spätestens jedoch unmittelbar vor der Hochzeit vollzogen wurde und das Ende der adoleszenten Lebensphase markierte. ${ }^{77}$ In Anlehnung an Victor Turners Weiterentwicklung des Initiationsansatzes des französischen Anthropologen Arnold van Gennep ${ }^{78}$ spricht Stannek von der Kavalierstour als einer "liminalen Phase im Leben adliger junger Männer"79 Liminal bezeichnet dabei nach Turner den „Status des Initianden während der sogenannten Umwandlungsphase", so Stannek. Die Kavaliere durchlebten bei einer erfolgreichen Tour eine Verwandlung, bei der der Ausschluss aus der Welt der Erwachsenen wichtig war, damit sie „die Symbole und Zeichen ihrer Kultur begriffen." ${ }^{\text {"80 }}$ Auch Wolfgang Treue geht vom Initiationscharakter von frühneuzeitlichen Reisen in der Phase der Adoleszenz aus. Diese boten, so schreibt er, Jugendlichen und jungen Erwachsenen „Freiräume, wie sie sie weder im elterlichen Haus noch in der streng geordneten Welt der Erwachsenen erleben konnten, in die sie meist bald nach der Rückkehr eintraten." ${ }^{81}$

Hier klingt bereits an: Zu dieser liminalen Phase gehörte allem Anschein nach auch ein gewisses Maß an Normverstößen. Dies zeigt die von Stannek erwähnte relative Toleranz der Gesellschaft gegenüber diesen „Kavaliersdelikten“ - laut Stannek wäre dies der passende Begriff, hätte es ihn schon im 17. Jahrhundert gegeben. Den jungen Adeligen wurde "mangelhafte Affektkontrolle" attestiert, wodurch ihr nonkonformes Verhalten einigermaßen entschuldigt war. ${ }^{82}$

Dass die Kavaliere auf Reisen also nicht ihre ganze Zeit mit Studien und dem Erlernen höfischer Umgangsformen zubrachten, wird vor allem bei diesem letzten Punkt deutlich. Keller etwa unterteilt die Aktivitäten auf der Kavalierstour in ein Bildungs-, Besuchs- und Besichtigungsprogramm. Letzteres umfasste Sehenswürdigkeiten aller Art. Keller nennt unter anderem Gärten und Paläste, Kirchen, Festungen, Werkstätten, Gemäldesammlungen und Raritätenkabinette.

Zu beachten ist dabei: Nicht das, was die Reisenden persönlich interessierte, suchten sie auf, sondern das, was Reiseführer oder Herren von Stand ihnen nahelegten. Die Besichtigung wiederum verlief nach stark vorgeprägten Wahrnehmungsmustern ab

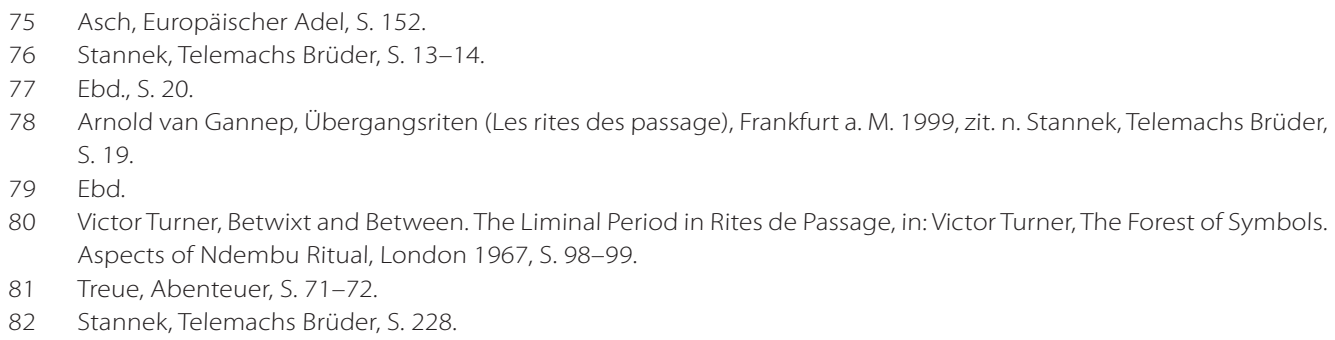


- Keller spricht hier von den "Grenzen des Blicks" - sodass kultureller Transfer nur sehr selektiv vonstatten ging. Das Thema der Fremdwahrnehmung soll später noch näher beleuchtet werden.

Darüber hinaus war auch der Kontakt zu Gleichaltrigen, anderen jungen Kavalieren auf Reisen, ein wesentlicher Bestandteil des Zeitvertreibs auf Tour. Dass es dabei vor allem "beim geselligen Trinken und bei den gewalttätigen Auseinandersetzungen" zu "mehr oder weniger ritualisierten Übertretungen der Normen höfischen Verhaltens" kam, bescherte der Phase später adeliger Adoleszenz in der älteren Forschung den Namen "Flegeljahre", so Stannek.83 Auf die relative Toleranz gegenüber nonkonformen Verhaltens der Kavaliere, deren Tour erst dazu beitragen sollte, Affektkontrolle zu erlernen, deren Beherrschung also noch nicht vorausgesetzt wurde, ${ }^{84}$ wurde bereits hingewiesen. In Bezug auf den Alkoholkonsum, der den Kavalieren zum einen als „Vehikel sozialer Integration", andererseits als Ventil und Ersatz für kriegerische Auseinandersetzungen, um die gruppeninternen Hierarchien festzulegen, diente, sei hier noch erwähnt, dass entgegen den strengen Ermahnungen der verhaltensanleitenden Literatur exzessives Trinken durchaus "mit den Maximen der höfischen Männlichkeit" zu vereinbaren war. Wirkliche Zurückhaltung diesbezüglich gab es in der Praxis des höfischen Alltags nur bei bestimmten zeremoniellen Anlässen, so Stannek. ${ }^{85}$

Welchen Stellenwert die Kavalierstour im Leben junger Adeliger hatte, geht daraus hervor, dass die Erziehung der Söhne adeliger Familien schon früh in weiten Teilen eine Vorbereitung auf die Tour darstellte. So wurden etwa Fremdsprachenkenntnisse vermittelt, Geographie- und Politikunterricht erteilt und Reiseberichte und Topographie als Lektüre bestimmt. Beaufsichtigt wurde der Unterricht dabei von erwachsenen, bürgerlichen Männern, den sogenannten Tutoren oder Hofmeistern. ${ }^{86}$ Da diese als Begleiter auch bei der Kavalierstour selbst eine wesentliche Rolle spielten und in der überwiegenden Zahl der Fälle die Reiseberichte verfassten, sollen sie nun genauer in den Blick genommen werden.

\section{$2.4 \quad$ Tutoren}

Nicht nur für die jungen Adeligen erfüllte die Kavalierstour wichtige Funktionen, auch Bürgerliche konnten davon profitieren. Als Begleiter der Kavaliere bot ihnen die Reise die Möglichkeit, sich selbst fortzubilden, eventuell angesehene Universitäten zu besuchen und dort zu promovieren. ${ }^{87}$ Häufig eröffnete ihnen dies Karrieremöglichkeiten in Verwaltung und Diplomatie. ${ }^{88}$

83 Stannek, Telemachs Brüder, S. 215.

84 Ebd., S. 216-218.

85 Ebd., S. 221-222.

86 Ebd., S. 33-39.

87 Ridder-Symoens, Mobilität, S. 346.

88 Stannek, Telemachs Brüder, S. 211. 
Mentor, paedagogus, Präzeptor, Hauslehrer, Tutor, Hofmeister - die Bezeichnungen für diese Begleitpersonen waren vielfältig und sind es auch in der heutigen Forschung noch, ${ }^{89}$ wobei es durchaus Unterschiede gab und gibt. Der Begriff Hofmeister beschränkte sich auf die Erzieher der Söhne von Regentenfamilien, also kaiserlicher, königlicher oder fürstlicher Dynastien - nicht zu verwechseln mit dem Amt des Hofmeisters an denselben Höfen, der sich um die Hauswirtschaft des Regenten kümmerte. Die Söhne fürstlicher Familien reisten auf ihrer Kavalierstour also in Begleitung ihres persönlichen Hofmeisters, der häufig ebenfalls adeliger Abstammung war, wohingegen mehrere Söhne reichsgräflicher Familien sich häufig einen Tutor bzw. Präzeptor, Mentor, paedagogus oder Hauslehrer bürgerlicher Abstammung teilten. ${ }^{90}$

Eines war den Begleitern unterschiedlicher Herkunft jedenfalls gemein - sie hatten auf der Tour keine leichte Aufgabe. Zum einen lag das an der Übergangsphase vom Jugendlichen zum Erwachsenen, in der sich ihre Schützlinge befanden. Autorität durchzusetzen war da keine leichte Aufgabe. Zum anderen wollten auch die Familien der jungen Adeligen ein Wörtchen mitreden. Deren Wünsche standen nicht selten im Widerspruch zu den pädagogischen Vorstellungen der Tutoren. Im Konfliktfall zogen letztere stets den Kürzeren. Die Vollmacht, die ihnen der Familienvater im Hinblick auf die Erziehung der Söhne übertrug, war in der Praxis wenig schlagkräftig. ${ }^{91}$

Unterwegs waren die Hofmeister und Tutoren für die Zusammenstellung des Reise-, Besichtigungs- und Besuchsprogrammes verantwortlich, ${ }^{92}$ waren also bereits in die Reisevorbereitungen intensiv involviert. ${ }^{93}$ Zudem überwachten sie den Bildungserfolg und das Verhalten ihrer Zöglinge und behielten die Finanzen im Überblick. ${ }^{94}$ Außerdem zählte es zu ihren Aufgaben, den Vater bzw. Vormund über die Reise der Söhne zu informieren, was in zahlreichen Briefen geschah. Nach Abschluss der Reise waren auch sie, die Tutoren und Hofmeister, es, die den umfassenden Reisebericht verfassten, der für die höfische Öffentlichkeit konzipiert war und zum Teil gedruckt wurde. ${ }^{95}$ Der im zweiten Teil der Arbeit analysierte Reisebericht etwa stammt aus der Feder Johann Georg Keyßlers, der in Diensten des Grafen Andreas Gottlieb von Bernstorff, Minister des Kurfürstentums Braunschweig-Hannover, stand und mit dessen Enkeln Andreas Gottlieb und Johann Hartwig Ernst ab 1727 mehrere Reisen unternahm, unter anderen ab 1729 jene Reise, die Gegenstand der Untersuchung ist. Von ihr berichtete Keyßler seinem Herren, Graf Andreas Gottlieb von Bernstorff, in 99 Briefen, die in ihrer Gesamtheit als Reisebericht veröffentlicht wurden. 


\subsection{Frühneuzeitliche Reiseliteratur und die Methodisierung des Reisens}

Mit dem Aufkommen der bereits erwähnten humanistischen Bildungsreise am Beginn der Neuzeit sind zwei weitere Entwicklungen verknüpft, die auch untereinander in Verbindung stehen: Die Methodisierung des Reisens begann und neue Formen von Reiseliteratur entstanden. ${ }^{96}$

Das Zeitalter der Entdeckungen bescherte Europa einen Mentalitätswandel: Das „Fremde“ wurde nun neu bewertet, nicht mehr bloß als das „Auszugrenzende oder zu Vereinnahmende begriffen", sondern positiv als etwas Bereicherndes wahrgenommen. ${ }^{97}$ Damit änderte sich auch die Sicht auf das menschliche Wissen, das nicht länger als abgeschlossen, sondern als erweiter- und verbesserbar galt, was wiederum eine positivere Bewertung der menschlichen Neugierde brachte, die im Mittelalter als schweifender und unstabiler Geisteszustand abgelehnt, nun aber als Schlüssel zu neuem Wissen betrachtet wurde. ${ }^{98}$

Die reisenden Humanisten sahen sich selbst und ihre Reisen im Dienste der Allgemeinheit und machten den Wissensfortschritt, im Sinne von Wissen für die Allgemeinheit, neben der Persönlichkeitsbildung zum zweiten zentralen Zweck ihres Unterwegsseins. ${ }^{99}$ Damit die Reise des Einzelnen zum allgemeinen Wissenserwerb beitragen konnte, wurden ihm Ratschläge mit auf den Weg gegeben - und zwar nicht mehr privat und überwiegend mündlich, sondern standardisiert und vor allem schriftlich und öffentlich. Kurzum: Die Methodisierung des Reisens hatte begonnen und dabei entstand eine neue literarische Gattung: der "Reiseratgeber". ${ }^{00}$ Diese Ratgeber, auch Apodemiken genannt, enthielten neben anderen Ratschlägen auch Anweisungen zum richtigen Umgang mit Unbekanntem und sollten die Aufmerksamkeit und die Wahrnehmung lenken. Dem Reisenden wurde vermittelt, wie er Beobachtungen macht, wie er Fragen stellt, wie er Erkenntnisse festhält, diese ordnet und auswertet und schließlich schriftlich fixiert. ${ }^{101}$ Die humanistische Bildungsreise und der damit verbundene Beginn der Methodisierung des Reisens führte, wie aus diesem Punkt ersichtlich wird, also auch zum Wandel einer bereits bestehenden literarischen Gattung: der des Reiseberichts.

Reiseberichte - die Peter Brenner grob als „sprachliche Darstellung authentischer Reisen“ definiert, wobei er allerdings einen „breite[n] Spielraum zwischen Authentizität und Fiktionalität" diagnostiziert ${ }^{102}$ - dienten im Mittelalter, entsprechend der damals vorherrschenden Vorstellung , ,alles zu Wissende“ sei „durch die Antike, vornehmlich die Bibel, bereits festgestellt", nicht der Generierung neuen Wissens. ${ }^{103}$ Zudem waren sie

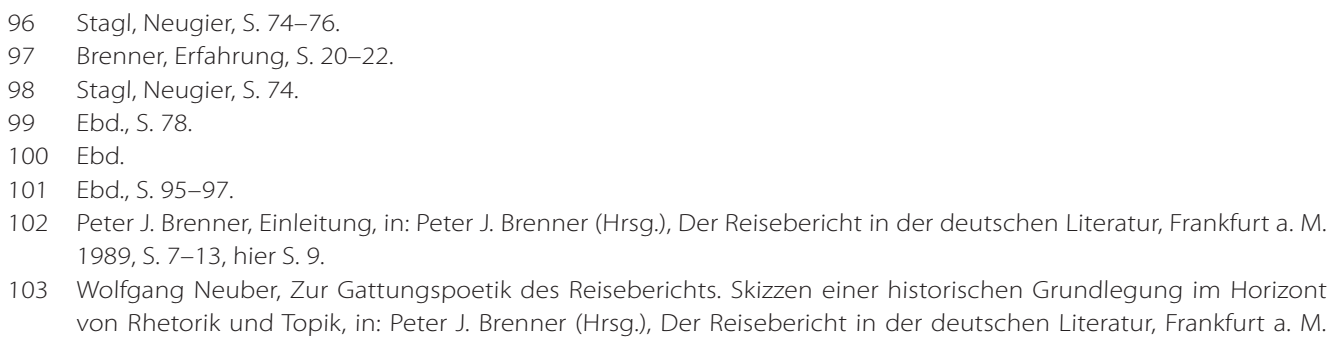

103 Wolfgang Neuber, Zur Gattungspoetik des Reiseberichts. Skizzen einer historischen Grundlegung im Horizont von Rhetorik und Topik, in: Peter J. Brenner (Hrsg.), Der Reisebericht in der deutschen Literatur, Frankfurt a. M. 
nach Disziplinen organisiert, sodass die Berichte von Pilgern zur theologischen Literatur gezählt wurden, die Berichte „weltlicher", utilitärer Reisen zur Gattung der navigatio, die Themen aus Seefahrt, Handel und Erdkunde vereinte. ${ }^{104}$

In der Frühen Neuzeit jedoch positionierte sich der Reisebericht im „Vorhof der Wissenschaft", wie Stagl schreibt, und leistete damit einen grundlegenden Beitrag zur "Eingliederung von Erfahrungswissen in den Bereich systematischen, gesicherten Wissens."105 Zudem war der frühneuzeitliche Reisebericht nicht mehr Fach-, sondern Sachprosa, wie Neuber anmerkt, ${ }^{106}$ was laut Stagl bedeutet, dass er in seinen Themen vielseitiger wurde und fortan Platz für unterschiedliche Erfahrungen bot. ${ }^{107}$

Die durch die apodemische Literatur vorstrukturierte Erfahrung von Fremdheit schlug sich in den Reiseberichten in Form einer so weit wie möglich gehenden Ausschaltung des persönlichen Moments und dem Bemühen um einen trockenen und sachlichen Stil nieder. Der um innere Selbstkontrolle bemühte Reisende verfolgte damit das Ideal des Objektivismus. ${ }^{108}$

Die bereits angesprochene Neubewertung des „Fremden“ und das große Interesse an unbekannten Weltgegenden führten in Europa zu einer großen Nachfrage an Reiseberichten, die ihren Beitrag dazu leisteten, dass nicht nur Europa zunehmend in der Welt präsent war, sondern auch das "Fremde“ in Europa allgegenwärtig war. ${ }^{109}$ Das folgende Kapitel widmet sich nun eingehender dem Phänomen der Fremdheitswahrnehmung in der Frühen Neuzeit.

\section{Das „Fremde“ in der Frühen Neuzeit}

Allen frühneuzeitlichen Reisen war, so schreibt Wolfgang Treue, ein gewisses Maß an Fremdheitserfahrung gemeinsam. Der Grad der empfundenen Fremdheit und die Intensität der Fremdheitserfahrung waren nicht zwingend von der zurückgelegten Entfernung abhängig. Die „Fremde“ konnte demnach auch in der unmittelbaren Umgebung des Ausgangspunktes beginnen. ${ }^{110}$ Wenn man allerdings bedenkt, dass sich menschliche Gefühle niemals mit Sicherheit bestimmen lassen und es umso schwieriger ist, die Gefühls- und Erfahrungswelt von Menschen zu rekonstruieren, die vor einigen hundert Jahren gelebt haben, worauf im Übrigen auch Treue hinweist, ${ }^{111}$ ist diese Aussage durchaus mit Vorsicht zu genießen. Für die Untersuchung von Reiseberichten im Hinblick auf Fremdheitserfahrungen bedeutet dies, dass in keinem Fall mit hundertprozentiger Gewissheit festgestellt werden kann, ob die geschilderten Erlebnisse mit empfundener Fremdheit verbunden waren.

1989, S. 50-67, hier S. 56.

104 Stagl, Neugier, S. 74.

105 Ebd., S. 74.

106 Neuber, Gattungspoetik, S. 57.

107 Stagl, Neugier, S. 74.

108 Ebd., S. 107.

109 Treue, Abenteuer, S. 17-19.

110 Ebd., S. 7.

111 Ebd., S. 141. 
Treue nennt allerdings einige Indizien, die seiner Ansicht nach auf Fremdheitserfahrungen hinweisen. Dazu zählt beispielsweise ein Phänomen, das hier abgekürzt als Sprachlosigkeit bezeichnet werden soll. Darunter ist die, wie Treue es beschreibt, mitunter sehr unbeholfene und umständliche Beschreibung von Dingen, für die es dem Verfasser des Berichts an Begrifflichkeiten und Konzepten fehlt, zu verstehen. Diese tritt sowohl bei der Beschreibung von menschlichen Artefakten wie Bauwerken, sowie bei exotischen Tieren und landschaftlichen Phänomenen auf. Als Beispiel für letztere nennt Treue etwa die Umschreibung der Wüste als „Sandmeer". Diese (partielle) Sprachlosigkeit in Anbetracht unbekannter Dinge ist, wie daraus deutlich wird und auch Treue schreibt, daher in der Regel mit Versuchen zu ihrer Überwindung verbunden. ${ }^{112}$

Weitere Hinweise auf ein Gefühl von Fremdheit liefern laut Treue Zahlenangaben, sowohl sehr ungenaue, grob übertriebene, mit denen lediglich vermittelt werden sollte, dass etwas sehr viel, sehr groß und dergleichen war, als auch sehr exakte, etwa bei der Vermessung der heiligen Stätten in Jerusalem, die nicht nur der Erinnerung und der Vermittlung der Topographie dienten, sondern auch als „mystische Komponente der Aneignung des Heilsgeschehens" betrachtet wurden. ${ }^{113}$ Auch Vergleiche weisen laut Treue auf Fremdheitserfahrungen hin, ebenso wie Superlative, Verweise auf "die Unmöglichkeit der Beschreibung“ und der "ausdrückliche Verzicht auf eine solche unter Hinweis auf Zahl oder die Qualität bereits existierender Schilderungen."

Die Analyse eines Reiseberichts in vorliegender Arbeit soll auch nach Hinweisen auf Fremdheitserfahrungen suchen, dabei wurde jedoch Treues Liste möglicher Indizien wie folgt erweitert: Als Hinweis auf eine Fremdheitserfahrung wird außerdem die Erwähnung bestimmter Details gewertet. Wenn etwa der Verfasser des Berichts in den untersuchten Abschnitten kaum auf kulinarische Belange eingeht, können jene seltenen Fälle, in denen er es tut, als Hinweise darauf betrachtet werden, dass er das erwähnte Detail als besonders oder sogar als fremd betrachtete. Dieser Hinweis soll bei der Untersuchung abgekürzt mit dem Begriff Erwähnung bezeichnet werden. Außerdem werden alle Stellen, in denen der Verfasser etwas explizit als „fremd“, "besonders" oder ihm bis dahin unbekannt bezeichnet, als Hinweise auf Fremdheitserfahrungen gewertet, die Bezeichnung für diesen Hinweis lautet Explikation. Zudem wird Treues Kriterium der exakten Zahlenangaben allgemeiner formuliert: Generell sollen sehr detaillierte Beschreibungen als Hinweise auf Fremdheitserfahrungen gewertet werden.

Der Schwerpunkt der Untersuchung soll aber auf der allgemeinen Frage liegen, welche kulturellen Themen und Phänomene der Verfasser anspricht - erst in einem nächsten Schritt soll festgestellt werden, ob sich dabei Hinweise auf Fremdheitserfahrungen finden.

Der Kulturbegriff ist zweifelsohne nicht unumstritten. Eine ausführliche Diskussion des Begriffs würde den Rahmen dieser Arbeit sprengen. Für die Analyse des Reiseberichts scheint es dennoch sinnvoll, am Kulturbegriff festzuhalten und zwar im Sinne eines

112 Treue, Abenteuer, S. 141-143 und S. 156.

113 Ebd., S. 156-157. 
anthropologischen Kulturverständnisses, das Kultur und Natur gegenüberstellt. Demzufolge soll in der Analyse eine Zuordnung kultureller Themen und Phänomene entlang von vierzehn Kategorien erfolgen.

Vier dieser Kategorien werden dabei von Treue übernommen. Im Hinblick auf die Untersuchung von Fremdheitserfahrungen sind sie vielversprechend, da Treue schreibt, dass Fremdheitserfahrungen frühneuzeitlicher Reisender in diesen Bereichen besonders häufig bzw. besonders intensiv waren. Diese sind: Religion und Konfession, gesellschaftliche Ordnung (insbesondere die Rolle von Frauen in der Gesellschaft sowie das Verhältnis der Geschlechter), Nahrungsmittel und Tischsitten sowie "nationale bzw. ethnische Vorurteile."114 Letzter Punkt soll kurz näher beleuchtet werden, u. a., um Begriffe wie Vorurteil und Stereotyp zu klären und zu unterscheiden.

Um die komplexe Realität ordnen und bewältigen zu können, sind Menschen auf Typisierungen angewiesen, doch nicht jede Verallgemeinerung ist zugleich auch ein Stereotyp. Bei Stereotypen, so die Historiker_innen Hans und Eva Hahn, kommt notwendigerweise eine starke emotionale Komponente hinzu, was sie resistent gegen Kritik und somit nicht falsifizierbar macht. ${ }^{115}$ Laut Hahn und Hahn handelt es sich bei einem Stereotyp also um eine Aussage, genauer ein positives oder negatives Werturteil, hinter dem eine starke Überzeugung steht und auf Menschen und Menschengruppen sowie auf Beziehungen zwischen Menschen und Menschengruppen bezogen und dabei stark generalisierend ist. ${ }^{16}$ Im allgemeinen Sprachgebrauch wie in der Forschungsliteratur wird nicht immer klar zwischen den Begriffen Stereotyp und Vorurteil unterschieden. Laut Edmund Lipiansky besteht der Unterschied jedoch darin, dass es sich bei Vorurteilen um Vorstellungen von Menschen oder Menschengruppen handelt, die existieren, ohne dass der Mensch bzw. die Menschen, auf die sie bezogen sind, vom Subjekt jemals getroffen wurden. Stereotype hingegen können sich „auch auf konkrete Erfahrungen gründen". ${ }^{117}$ Gordon Allport wiederum bringt das Vorurteil in Verbindung mit Handlungen und bezeichnet es daher als „ablehnende oder feindselige Haltung gegen eine Person, die zu einer Gruppe gehört, einfach deswegen, weil sie zu dieser Gruppe gehört und deshalb dieselben zu beanstandenden Eigenschaften haben soll, die man dieser Gruppe zuschreibt."118

Auffallend bei diesen Definitionen ist jedenfalls, dass anhand der Analyse des Reiseberichtes nicht festgestellt werden kann, ob die darin enthaltenen verallgemeinernden Aussagen lediglich Verallgemeinerungen, Stereotype oder Vorurteile sind. Statt von "nationalen bzw. ethnischen Vorurteilen" soll in der Untersuchung daher von "Aus-

114 Treue, Abenteuer, S. 185-186.

115 Hans Henning Hahn/Eva Hahn, Nationale Stereotypen. Plädoyer für eine historische Stereotypenforschung, in: Hans Henning Hahn/Stephan Scholz (Hrsg.), Stereotype, Identität und Geschichte. Die Funktion von Stereotypen in gesellschaftlichen Diskursen (Mitteleuropa - Osteuropa. Oldenburger Beiträge zur Kultur und Geschichte Ostmitteleuropas 5), Frankfurt a. M. 2002, S. 17-56, hier S. 22.

116 Hahn/Hahn, Nationale Stereotypen, S. 20-21.

117 Edmund Marc Lipiansky, Die Komplexität der Vorstellung vom Anderen, in: Hans Nicklas/Burkhard Müller/ Hagen Kordes (Hrsg.), Interkulturell denken und handeln. Theoretische Grundlagen und gesellschaftliche Praxis, Frankfurt a. M.-New York 2006, S. 117-120, hier S. 117.

118 Gordon W. Allport, Die Natur des Vorurteils, in: Klaus Alheim (Hrsg.), Die Gewalt des Vorurteils. Eine Textsammlung (Politik und Bildung 44), o.O. 2007, S. 40-59, hier S. 42. 
sagen zu Charaktereigenschaften von Gruppen" die Rede sein. Der Begriff Nationalcharakter wird bewusst vermieden. Das liegt nicht daran, dass es zur damaligen Zeit keine Vorstellungen dazu gegeben hätte. Laut Treue, Maurer und Schulze ist die Idee von Nationalcharakteren um einiges älter als die von Nationalstaaten. Schulze sieht Ursprünge im Mittelalter, Maurer spricht gar von antiken Wurzeln. Allerdings bemerkt Treue, dass derartige Vorstellungen bis ins Spätmittelalter wohl kaum relevant waren. ${ }^{119}$ Der Grund für den Verzicht auf den Begriff Nationalcharakter liegt viel eher darin, dass in der Untersuchung auch Aussagen zu kleineren Einheiten als Nationen betrachtet werden, etwa regionale Gruppen wie Sayovarden und Piemonteser, sodass der Begriff Nationalcharakter irreführend wäre.

Neben den bereits erwähnten vier Kategorien soll der Text noch hinsichtlich folgender anderer untersucht werden: 5) "Staatliche" Organisation (Verwaltung, Recht, Militär, Staats- und Regierungsform etc.), 6) Kleidung/Tracht, 7) Bildung, Wissenschaft und Technik, 8) Bauwerke, Architektur und bildende Kunst, 9) Brauchtum, Sitten und Gewohnheiten, 10) Sprache, 11) Wirtschaft und Finanzen, 12) historische Begebenheiten, Anekdoten, Erzählungen, Sagen und Legenden, 13) Literatur, 14) Musik.

\section{Analyse}

Bei der untersuchten Quelle handelt es sich um einen Reisebericht von Johann Georg Keyßler, zum Zeitpunkt der Reise im Dienst des ersten Staatsministers des Herzogtums Braunschweig-Lüneburg, Andreas Gottlieb von Bernstdorff. Der Bericht umfasst die zwischen 1729 und 1731 besuchten Stationen der Reise, die Keyßler als Hauslehrer der beiden Enkel seines Dienstgebers, Johann Hartwig Ernst und Andreas Gottlieb, bereiste. ${ }^{120}$

Untersucht wird allerdings nicht der gesamte Bericht, da dessen Umfang - der Bericht ist unterteilt in 99 Briefe und umfasst ohne Anhang 1.490 Seiten - den Rahmen der Seminararbeit sprengen würde. Stattdessen wurde eine Auswahl getroffen, die die geographische Bandbreite der Reise möglichst gut abdecken soll. Eine detaillierte Übersicht über die Stationen der Reise findet sich im Inhaltsverzeichnis des Berichts. ${ }^{121} \mathrm{Hier}$ soll nur die getroffene Auswahl näher umrissen werden. Diese umfasst die Briefe mit den Nummern 5-8, 21-34, 36 und 99. Diese behandeln folgende Stationen der Reise: Grafschaft Tirol (inklusive Innsbruck), Erzbistum Salzburg, kurfürstliche Residenzstadt München, Republik Genf, Königreich Sardinien, Herzogtum Mailand und Herzogtum Lothringen. Die Auswahl folgte dem Anspruch, Beschreibungen aus möglichst vielen der bereisten geografischen Großregionen in die Analyse aufzunehmen.

119 Treue, Abenteuer, S. 203; Maurer, Nationalcharakter, S. 49; Winfried Schulze, Die Entstehung des nationalen Vorurteils. Zur Kultur der Wahrnehmung fremder Nationen in der europäischen Frühen Neuzeit, in: Wolfgang Schmale/Reinhard Stauber (Hrsg.), Menschen und Grenzen in der Frühen Neuzeit (Innovation. Bibliothek zur Neueren und Neuesten Geschichte 2), Berlin 1998, S. 23-49, hier S. 25.

120 Gottfried Schütze, Vorrede, in: Johann Georg Keyßler, Neueste Reisen durch Deutschland, Böhmen, Ungarn, die Schweiz, Italien und Lothringen [...], Hannover 1751, S. I-XVI, hier S. VI-IX, [https://books.google.at/books/about/ Johann_Georg_Keysslers_Neueste_Reisen_du.html?id=W9AWAAAAQAAJ\&redir_esc=y], eingesehen 24.8.2017; Winfried Siebers, Johann Georg Keyßler und die Reisebeschreibung der Frühaufklärung, Würzburg 2009, S. 25-27.

121 Keyßler, Neueste Reisen. 
Zunächst erfolgt eine äußere und innere Kritik der Quelle. Daran schließt eine qualitative und quantitative Inhaltsanalyse des Reiseberichtes nach Philipp Mayerings Modell einer strukturierenden Inhaltsanalyse, genauer: gemäß seines Modells inhaltlicher Strukturierung, an. ${ }^{122}$ Diese wird wiederum in zwei Durchgänge unterteilt. Im ersten Durchlauf werden zunächst alle Textstellen der getroffenen Auswahl kodiert, die sich mit kulturellen Themen beschäftigen - das vierzehnteilige Kategoriensystem hierzu wurde bereits im vorhergehenden Kapitel vorgestellt. Anschließend wird in einem quantifizierenden Vorgang festgestellt, wie viel Raum die jeweiligen Kategorien in der Auswahl einnehmen. Dabei werden sowohl die gesamte Zeilenzahl der Kategorie, verglichen mit der Gesamtzeilenzahl kultureller Themen (bei Mehrfachkodierung werden die Zeilen nur einmal gezählt), als auch die Streuung der einzelnen Kategorien, also die Häufigkeit ihres Vorkommens in der Textauswahl, gegliedert nach Briefen, sowie die Anzahl der Fundstellen pro Kategorie berücksichtigt. Im zweiten Durchlauf werden die kodierten Textstellen auf Hinweise hinsichtlich Fremdheitserfahrungen untersucht.

\subsection{Quellenkritik}

Der Reisebericht Johann Georg Keyßlers erschien erstmals 1740 (Band 1) und 1741 (Band 2) in Hannover. Beim untersuchten Text handelt es sich um eine Auflage aus dem Jahr 1751, ebenfalls in Hannover erschienen.

Geboren wurde der Verfasser des Berichts, Johann Georg Keyßler, am 13.4.1693 in Thurnau im heutigen Oberfranken, ein kleines Territorium unter der Herrschaft der reichsunmittelbaren Grafen von Giech, das bis 1796 bzw. 1806 seine Selbstständigkeit gegenüber Annexionsversuchen der Markgrafschaft Brandenburg-Kulmbach und des Bistums Bamberg behauptete. ${ }^{123}$ Sein Vater, Johann Georg Keyßler (1658-1720), war Hofrat und Archivar der Grafen von Giech. Nach Abschluss der Schulausbildung begann der junge Keyßler im Jahr 1711 in Halle ein Studium der Rechtswissenschaften. ${ }^{124}$ Im Nebenfach studierte er germanische Altertumskunde, außerdem beschäftigte er sich laut Siebers intensiv mit „Philosophie, Theologie, alter Geschichte und den Sprachen Englisch, Französisch, Italienisch, Spanisch, Griechisch, Latein und Hebräisch sowie mit den ,schönen Wissenschaften'.'125 Nach anderthalb Jahren brach Keyßler aus gesundheitlichen Gründen das Studium ab, kehrte aber schon kurz darauf, als Unterhofmeister der Grafen Christian Carl und Carl Maximilian von Giech, nach Halle zurück ${ }^{126}$ - laut Siebers der Ausgangspunkt des „typischen Berufsweg[s] der in den Humaniora ausgebildeten Intelligenzschicht des 18. Jahrhunderts."127 Ab Juli 1713 unternahm Keyßler eine Bildungsreise mit den jungen Grafen, ${ }^{128}$ im Anschluss daran, im Jahr 1716, wurde Keyßler Hauslehrer von Johann Hartwig Ernst und Andreas Gottlieb von Bernstorff in

\footnotetext{
122 Philipp Mayering, Qualitative Inhaltsanalyse, Weinheim-Basel 2012.

123 Siebers, Keyßler, S. 20.

124 Ebd., S. 21

125 Ebd.

126 Schütze, Vorrrede, S. V; Siebers, Keyßler, S. 23.

127 Siebers, Keyßler, S. 23.

128 Schütze, Vorrede S. V; Siebers, Keyßler, S. 24.
} 
Hannover. ${ }^{129}$ Die Familie Bernstorff hatte in ihrem Familienstatut festgelegt, dass die häusliche Erziehung mit Vollendung des zwölften Lebensjahres abgeschlossen und daran anschließend eine Bildungsreise unternommen werden sollte, die mit einem Universitätsbesuch begann. ${ }^{130}$ Daher reiste Keyßler mit den Brüdern im Jahre 1727 nach Tübingen, wo sie die Universität und die Ritterakademie besuchten. Im Frühjahr 1729 starteten sie schließlich die Reise, ${ }^{131}$ deren Verlauf Keyßler in seinem in 99 Briefen unterteilten Bericht festhielt.

Adressat der Briefe war zum einen Keyßlers Dienstgeber, Andreas Gottlieb von Bernstorff, zum anderen, da der Bericht wie viele andere dieser Zeit später gedruckt und veröffentlicht wurde, kann auch eine breitere Öffentlichkeit als Adressat betrachtet werden.

Somit kann von einer zweifachen Absicht hinter Keyßlers Bericht ausgegangen werden. Zum einen dienten die Briefe dazu, den Dienstgeber über den Fortgang der Reise zu unterrichten, zum anderen im Sinne frühneuzeitlicher Reiseberichte einen Beitrag zurVermehrung des Wissens der Allgemeinheit zu leisten.

\subsection{Qualitative Inhaltsanalyse}

\subsubsection{Durchlauf 1: Behandlung kultureller Themen}

Gemäß Mayerings Modell der inhaltlichen Strukturierung wurden zunächst die Analyseeinheiten festgelegt. Bei diesem ersten Durchlauf waren Auswertungseinheit und Kodiereinheit ident: Jede Passage des Reiseberichts, in der es um kulturelle Aspekte gemäß des festgelegten Kategoriensystems geht, wurden zugleich als Auswertungs-, als auch als Kodiereinheit betrachtet. Unter Kontexteinheit wurde dabei alles Material verstanden, das in Zusammenhang mit der jeweiligen Erwähnung eines oder mehrerer kultureller Aspekte steht.

Schritt zwei und drei von Mayerings Modell fielen bei dieser Analyse ebenfalls zusammen: Auf eine umfassende Diskussion der Strukturierungsdimension (der Begriff Kultur) wurde verzichtet, stattdessen wurden 14 Kategorien erstellt, wodurch gleichzeitig Schritt 3, die Erstellung des Kategoriensystems, erfüllt war, die dem Bereich der Kultur im Sinne eines anthropologischen Verständnisses des Begriffes zugeordnet werden können. Das 14-teilige Kategoriensystem wurde bereits vorgestellt. Schritt 4, die Formulierung von Definitionen, Ankerbeispielen und Kodierregeln, soll hier nicht näher ausgeführt werden.

Der anschließende Materialdurchlauf hat ergeben, dass die untersuchten Textstellen insgesamt 7.862 Zeilen zu kulturellen Themen enthalten, wobei bei Mehrfachkodierungen die Zeilen nur einmal gezählt wurden. Am häufigsten darunter waren Textstellen, die der Kategorie 8 (Bauwerke, Architektur und bildende Kunst) zugeordnet werden 
können. Diese umfassen in allen zwanzig untersuchten Briefen insgesamt 2.768 Textzeilen bei 58 Fundstellen. An zweiter Stelle steht Kategorie 5 („Staatliche“ Organisation) mit 1.834 Zeilen bei Fundstellen. Auf Platz drei steht die Kategorie 12 (Anekdoten etc.) mit 1.815 Zeilen bei 70 Fundstellen. Weiters in absteigender Reihenfolge: Kategorie 7 (Bildung, Wissenschaft und Technik) mit 811 Zeilen bei 34 Fundstellen, Kategorie 1 (Religion und Konfession) mit 701 Zeilen bei 37 Fundstellen, Kategorie 9 (Brauchtum, Sitten und Gewohnheiten) mit 678 Zeilen bei 44 Fundstellen, Kategorie 11 (Wirtschaft und Finanzen) mit 603 Zeilen bei 27 Fundstellen, Kategorie 3 (gesellschaftliche Ordnung) mit 543 Zeilen (davon 232 bezogen auf Frauen bzw. das Geschlechterverhältnis) bei 16 Fundstellen, Kategorie 4 (Charaktereigenschaften von Gruppen) mit 153 Zeilen bei 13 Fundstellen, Kategorie 2 (Nahrungsmittel und Tischsitten) mit 109 Zeilen bei 15 Fundstellen; Kategorie 6 (Kleidung und Tracht) mit 51 Zeilen bei 8 Fundstellen, Kategorie 13 (Literatur) mit 14 Zeilen bei einer Fundstelle, Kategorie 10 (Sprache) mit 9 Zeilen bei vier Fundstellen, Kategorie 14 (Musik) mit 0 Zeilen.

Die Streuung wiederum sieht folgendermaßen aus: Kategorie 5 (Platz 2 bei der Zeilenzahl) findet sich in den meisten der Briefe, nämlich in 15 von 20. Die Kategorien 8 (Platz 1 bei der Zeilenzahl) und 12 (Platz 3 bei der Zeilenzahl) sind beide in 12 von 20 Briefen vertreten. Die Kategorie 1 (Platz 5 bei der Zeilenzahl) findet sich in 11 von 20 Briefen, die Kategorie 9 (Platz 6 bei der Zeilenzahl) in 10 von 20 Briefen. Die Kategorien 2 (Platz 10 bei Zeilenzahl), 3 (Platz 8 bei Zeilenzahl), 7 (Platz 4 bei Zeilenzahl) und 11 (Platz 7 bei Zeilenzahl) sind in je 6 der 20 Briefe vertreten. Die Kategorie 6 (Platz 11 bei Zeilenzahl) in 6 von 20, Kategorie 4 (Platz 9 bei der Zeilenzahl) in 5 von 20, Kategorie 10 (Platz 13 bei Zeilenzahl) in 4 von 20, Kategorie 13 (Platz 12 bei Zeilenzahl) in 2 von 20 und die Kategorie 14 (Platz 14 bei Zeilenzahl) in keiner der 20 untersuchten Briefe.

\subsubsection{Durchlauf 2: Hinweise auf Fremdheitserfahrungen}

In Textstellen der Kategorie 1 fanden sich insgesamt zehn Hinweise auf Fremdheitserfahrungen, darunter acht Vergleiche, eine Explikation sowie eine Erwähnung. Daraus ergibt sich in Bezug auf diese Kategorie ein Verhältnis zwischen den Hinweisen auf Fremdheitserfahrungen und der Zeilenzahl von 1:70. Bei fünf der acht Vergleiche handelt es sich um implizite Vergleiche, die also auf einen Vergleich mit einer Idealvorstellung schließen lassen, häufig sind diese impliziten Vergleiche auch mit einer positiven oder negativen Wertung verbunden. Ein Beispiel für einen expliziten Vergleich findet sich etwa im siebten Schreiben (Salzburg) auf der Seite 42: „Die Wüsteneyen der Gebirge im Tirolischen gegen Trente und im Salzburgischen haben Gelegenheit gegeben, daß von alten Zeiten her die verfolgten Waldenser hie und da in solche abgelegene Thäler geflüchtet, und eine den Protestanten in vielen Dingen gleichkommende Glaubenslehre fortgepflanzet haben." ${ }^{132}$ Ein Beispiel für einen impliziten Vergleich stellt etwa Keyßlers Kritik an der Art der Begräbnisse der Turiner Bevölkerung im 30. Brief dar. ${ }^{133}$ 
Bei der zweiten Kategorie finden sich insgesamt zwölf Hinweise (vier Erwähnungen, sechs Vergleiche, eine Explikation, und eine detaillierte Beschreibung). Das Verhältnis zwischen Hinweisen und Zeilenzahl beträgt 6:50. Ein Beispiel, das als Erwähnung zählt und eine Explikation und zwei Vergleiche enthält, ist folgendes:

„In dieser Gegend bekommt man ein besonderes Gericht Fische von ganz kleinen Neunaugen zu essen. Diese werden nur einen bis zween Finger lang, und kaum so dick, als ein halber Federkiel. Der Geschmack ist gut, und die hiesigen Aerzte erlauben sie bey allen Krankheiten. Man fängt sie in dem HintensteinerSee, der unter das Amt Kuffstein gehöret, und habe ich sie sonst nirgends gefunden, als hier und zu Turin."134

Bei der Kategorie 3 sind es acht Hinweise (vier Erwähnungen, eine Explikation und drei Vergleiche), das Verhältnis zwischen Hinweisen und Zeilenzahl beträgt 1:67. Ein Beispiel, das zugleich als Erwähnung und als Vergleich zählt, betrifft die gesellschaftlichen Verhältnisse in Mailand, genauer, die Lebensweise der Frauen: „Die Damen könnten in Frankreich selbst kaum mit wenigerm Zwange leben, als sie allhier thun. [...] Das weibliche Geschlecht von geringerm Stande nimmt gleichfalls allhier Theil an vielen Freyheiten, die ihnen an andern Orten Italiens abgeschnitten sind."135

Bei der Kategorie 4 sind es zehn Hinweise (sechs Vergleiche, zwei Erwähnungen und zwei Explikationen). Das Verhältnis zwischen Hinweisen und Zeilenzahl beträgt 1:15. Ein Beispiel für einen Vergleich, der auch eine Explikation enthält, ist folgendes:

"Mein Herr! Von dem humeur und der Lebensart der Sayovarden habe ich demselben zu anderer Zeit schon Bericht abgestattet, und komme ich itzt zu den Piemontesern, von welchen ich keineswegs die Vergleichung oder Parallele, welche selbst ein in hiesigem Lande gebohrner neulich gemacht hat, behaupten will, nämlich: es verhielten sich beyde Nationen also gegen einander, daß unter zehn Piemontesern vielleicht ein ehrlicher Mann sey, und unter zehn Sayovarden vielleicht ein Taugenichts oder Betrüger. Dieses ist aber gewiß, daß die Piemonteser überaus listigen Verstand besitzen, und zu wünschen wäre, daß ihr fertiger und scharfsinniger Kopf allezeit zum Guten angewendet würde. Auch der Missbrauch desselben geschieht gemeiniglich mit sowohl ausgesonnenen Umständen und Intriguen, daß man sich über ihren fähigen Geist [...] verwundern muß."136

Bei der Kategorie 5 fand sich lediglich eine Erwähnung. Bei besagter Textstelle handelt es sich um die Beschreibung des Prozederes an der Grenze bei der Einreise in die Grafschaft Tirol. ${ }^{137}$ Das Verhältnis zwischen Hinweisen und Zeilenzahl beträgt hier 1:1.834. Bei der Kategorie 6 fanden sich sechs Hinweise (zwei Erwähnungen, ein Vergleich und drei detaillierte Beschreibungen). Das Verhältnis zwischen Hinweisen und Zeilenzahl beträgt 1:8. Ein Beispiel für einen Vergleich und eine Erwähnung ist folgen-

\footnotetext{
134 Keyßler, Neueste Reisen, S. 41 
des: „Das gemeine Bauernvolk zieh in Tirol gar elend auf, und sieht den Zigeunern nicht gar unähnlich." Die Untersuchung der siebten Kategorie ergab sieben Hinweise (zwei Erwähnungen, zwei Bezeichnungen, drei Vergleiche). Das Verhältnis beträgt 7:811. Bei Kategorie 8 fanden sich elf Hinweise (sieben Vergleich, zwei detaillierte Beschreibungen und eine Explikation). Das Verhältnis beträgt 11:2.768. Bei Kategorie 9 sind es fünf Hinweise (vier Erwähnungen sowie die einzige Sprachlosigkeit). Das Verhältnis beträgt 1:170. Die Sprachlosigkeit tritt beim Versuch der Beschreibung des bunten Treibens beim Karneval in Mailand auf. ${ }^{138}$ Die Untersuchung der zehnten Kategorie ergab insgesamt vier Hinweise auf Fremdheitserfahrungen (drei Erwähnungen und ein impliziter Vergleich). Das Verhältnis zwischen diesen Hinweisen und der Gesamtzeilenzahl in Bezug auf Kategorie 10 beträgt 4:9. Ein Beispiel für eine Erwähnung und zugleich einen nicht wertenden impliziten Vergleich bezieht sich auf die Eigenheit der Tiroler Aussprache am Beispiel des Schlosses Ambras: „Die Tiroler sprechen es Ombras aus, gleich wie sie Schwoz für Schwaz sagen." Bei Kategorie 11 sind es sieben Hinweise (drei Vergleiche, eine Erwähnung und drei detaillierte Beschreibungen). Letztere betreffen die Beschreibung der Münze Hall sowie die Salzbergwerke Reichenhall und Hallein. Bei Kategorie 12 fanden sich vier Hinweise (drei detaillierte Beschreibungen und ein Vergleich). Das Verhältnis beträgt 4:1.815. Bei den Kategorien 13 und 14 fanden sich keine Hinweise.

Tabelle 1: Ergebnisse der Inhaltsanalyse

\begin{tabular}{|c|c|c|c|c|c|c|}
\hline Kategorie & $\begin{array}{l}\text { Zeilenzahl } \\
(Z Z)\end{array}$ & $\begin{array}{c}\text { Fundstellen } \\
\text { FS }\end{array}$ & $\begin{array}{l}\text { Streuuung } \\
\text { (ST) }\end{array}$ & $\begin{array}{l}\text { Hinweise auf } \\
\text { Fremdheits- } \\
\text { erfahrungen } \\
\text { (HFE) }\end{array}$ & $\begin{array}{c}\text { Verhältnis } \\
\text { HFE/FS }\end{array}$ & Verhältnis HFE/ZZ \\
\hline 1 & 701 (5.) & $37(4)$. & $11 / 20$ (3.) & $10(3)$. & 0,27 (6.) & $1: 70=0,014(6)$. \\
\hline 2 & 109 (10.) & 15 (9.) & $9 / 20(5)$. & $12(1)$. & 0,8 (2.) & $6: 50=0,11$ (3.) \\
\hline 3 & $543(8)$. & $16(8)$. & $9 / 20(5)$. & $8(4)$. & $0,5(5)$. & $\begin{array}{c}1: 67=0,015(5 .) \\
\text { bzw. } 8: 320(0,025)\end{array}$ \\
\hline 4 & 153 (9.) & $13(10)$. & $5 / 20(7)$. & $10(3)$. & 0,76 (3.) & $1: 15=0,06(4)$. \\
\hline 5 & $1.834(2)$. & $28(6)$. & $15 / 20(1)$. & $1(9)$. & 0,035 (12.) & 1:1.834 (12.) \\
\hline 6 & 51 (11.) & $8(11)$. & $6 / 20$ (6.) & $6(6)$. & 0,75 (4.) & $1: 8=0,125$ (2.) \\
\hline 7 & 811 (4.) & $34(5)$. & $9 / 20(5)$. & $7(5)$. & 0,2 (8.) & $7: 811=0,008$ (8.) \\
\hline 8 & 2.768 (1.) & $58(2)$. & $12 / 20(2)$. & $11(2)$. & 0,18 (9.) & $\begin{array}{c}11: 2.768=0,004 \\
(10 .)\end{array}$ \\
\hline 9 & $678(6)$. & 44 (3.) & $10 / 20(4)$. & $5(7)$. & 0,11 (10.) & $5: 678=0,007$ (9.) \\
\hline 10 & 9 (13.) & $4(12)$. & 4/20 (8.) & $4(8)$. & $1(1)$. & $4: 9=0,444(1)$. \\
\hline 11 & $603(7)$. & 27 (7.) & $9 / 20(5)$. & $7(5)$. & 0,25 (7.) & $7: 603=0,01$ (7.) \\
\hline 12 & 1.815 (3.) & $70(1)$. & $12 / 20(2)$. & $4(8)$. & 0,057 (11.) & $\begin{array}{c}4: 1.815=0,002 \\
(11)\end{array}$ \\
\hline 13 & $14(12)$. & 1 (13.) & 2/20 (9.) & $0(10)$. & 0:1 (13.) & $0: 14$ (13.) \\
\hline 14 & $0(14)$. & $0(14)$. & $0 / 20$ (10.) & $0(10)$. & $0: 0$ (14.) & 0:0 (13.) \\
\hline
\end{tabular}


Insgesamt waren es 84 Hinweise auf Fremdheitserfahrungen, darunter 39 Vergleiche, 24 Erwähnungen, zehn detaillierte Beschreibungen, acht Explikationen und eine Sprachlosigkeit.

\subsubsection{Interpretation der Ergebnisse}

Wie sind die Ergebnisse dieser Untersuchungen nun zu interpretieren, vor allem auch in Hinblick auf die eingangs aufgestellten bzw. von anderen übernommenen und zu überprüfenden Thesen?

Die Ergebnisse in Bezug auf Kategorie 1, Religion und Konfession, zeigen, dass dieses Thema in etwas mehr als der Hälfte der Schreiben erwähnt wird und bei der Gesamtzeilenzahl im Mittelfeld liegt. Die Anzahl der Hinweise auf Fremdheitserfahrungen beträgt zehn, was verglichen mit der Häufigkeit der generellen Fundstellen dieser Kategorie einen Prozentsatz von 27 ergibt. Dies ist angesichts der von Treue aufgestellten Behauptung, die Konfrontation mit fremden Religionen stellten in der Frühen Neuzeit so etwas wie eine "Grenze der Offenheit" dar, weniger als erwartet. Daher muss auch die von Maurer aufgestellte These verworfen werden, dass in der Frühen Neuzeit konfessionelle oder religiöse Fremdheit sehr stark wahrgenommen wurden. ${ }^{139}$

Die Kategorie 2, die sich auf das Essen, die Nahrungsmittel und die Tischsitten bezieht, kommt in den ausgewerteten Textstellen verhältnismäßig selten vor. In Relation zu dieser Seltenheit ist die Häufigkeit von Hinweisen auf Fremdheitserfahrungen aber sehr hoch - bei 80 Prozent der Fundstellen fand sich auch ein Hinweis auf eine Fremdheitserfahrung. Dies entspricht auch Treues Einschätzung, dass das Essen nur bei wenigen Reiseberichten der Frühen Neuzeit eine zentrale Rolle spielt, bei fast allen aber gelegentlich Bemerkungen dazu zu finden sind „, die seinen hohen Stellenwert im Rahmen der Fremderfahrung verdeutlichen."140

Über die gesellschaftlichen Verhältnisse im Allgemeinen wird, verglichen mit anderen kulturellen Themen, eher selten berichtet. Über Frauen und das Geschlechterverhältnis im Speziellen geht es dabei nur in weniger als der Hälfte der Fälle. Auffallend ist allerdings, dass jene Stellen, die von Frauen handeln, fast zur Gänze auch Hinweise auf Fremdheitserfahrungen liefern. Treue dürfte somit mit seiner Behauptung richtig liegen, dass Frauen auf Reisen sehr genau beobachtet wurden. ${ }^{141}$ Aus dem Umstand, dass Frauen zwar selten erwähnt, in diesen seltenen Fällen aber sehr oft Hinweise auf Fremdheitserfahrungen zu finden sind, zu schlussfolgern, dass Frauen und damit auch jede Abweichung von Bekanntem sehr genau beobachtet und registriert wurden, ist durchaus plausibel.

Ridder-Symoens These jedoch, dass Reiseberichte „die Entwicklung nationaler Stereotypen und Vorurteile" förderten, kann mit den vorliegenden Ergebnissen nur bedingt bestätigt werden - verallgemeinernde Aussagen über den Charakter von Gruppen 
sind zwar durchaus vorhanden, aber verhältnismäßig selten, wodurch ihr Gewicht im großen Umfang des gesamten Berichtes infrage gestellt werden kann.

In Bezug auf die Kategorien 5, 8 und 12 ist auffällig, dass diese den größten Raum unter den kulturellen Themen in den untersuchten Textstellen einnehmen, sich darin aber kaum Hinweise auf Fremdheitserfahrungen finden. Es ist daher naheliegend zu vermuten, dass für den umfassend gebildeten und zu dem Zeitpunkt bereits weit gereisten Autor auch fremde Staatsformen wie die Republik, Besonderheiten der Architektur oder historische Ereignisse nichts völlig Unbekanntes waren, es aber trotzdem erwartet wurde, diese Themen in Reiseberichten zu erwähnen, der Verfasser also einem zur damaligen Zeit typischen Muster zur Erstellung von Reiseberichten folgte.

Auch Themen aus Wissenschaft, Bildung und Technik scheinen ihm selten fremd zu sein, am ehesten finden sich Hinweise darauf in seinen ausführlichen Beschreibungen komplizierter Ingenieurskunst, etwa in Bezug auf die Münzprägestätte in Hall in Tirol.

Interessant ist zudem, dass die Schilderungen von Bräuchen, Sitten und Gewohnheiten (durchschnittlich oft erwähnt), nur verhältnismäßig wenige Hinweise auf Fremdheitserfahrungen beinhalten - auch hier scheint es plausibel, dies auf Vorkenntnisse des Autors zurückzuführen.

Sprache und Kleidung jedoch, die Kategorien 10 und 6, werden zwar nur sehr selten beschrieben, wenn aber, dann finden sich in den meisten Fällen auch Hinweise auf Fremdheitserfahrungen. In Bezug auf die sprachlichen Aspekte, die er ausschließlich nur nennt, wenn etwas daran auffällig ist, ließe sich ein Hinweis auf sein philologisches Interesse, dem Keyßler, wie bereits erwähnt, in seinem Studium nachging, vermuten.

\section{Fazit}

In der Arbeit wurde dem Phänomen studentischer Migration in der Frühen Neuzeit am Beispiel der überwiegend adeligen Kavalierstour nachgegangen. Dabei stand im Fokus der Blick auf die Wahrnehmung kultureller Aspekte sowie damit verbundener Fremdheitserfahrungen der Reisenden. Konkret wurde anhand eines Reiseberichts untersucht, welche kulturellen Themen besonders beachtet wurden und ob sich bei der schriftlich fixierten Beobachtung derselben Hinweise auf Fremdheitserfahrungen finden. Dabei wurden mehrere Thesen überprüft. Zum einen die These, dass in der Frühen Neuzeit, die oftmals als „konfessionelles Zeitalter" bezeichnet wird, vor allem religiöse und konfessionelle Aspekte eine zentrale Rolle spielten und sich dies, verbunden mit Hinweisen auf Fremdheitserfahrungen, in den Reiseberichten niederschlug. AuBerdem wurde Hilde de Ridder-Symoens'These, frühneuzeitliche Reiseberichte hätten einen wesentlichen Beitrag zur Entstehung nationaler Vorurteile geleistet, überprüft. Ebenfalls auf ihre Gültigkeit in Bezug auf den ausgewählten Reisebericht wurden Wolfgang Treues Aussagen, Frauen seien auf den Reisen sehr genau beobachtet worden, Andersartigkeit in Bezug auf Religion wurde wenig toleriert und Fremdheit in Bezug auf Nahrung und Tischsitten wurde besonders stark empfunden, überprüft. 
Der Analyse vorangestellt wurde ein Theorieteil, in welchem zunächst mehrere Aspekte des Phänomens der Kavalierstour beleuchtet wurden. So wurde etwa auf die unterschiedlichen Vorläufer und Traditionslinien der Tour hingewiesen sowie auf die Rolle einer Veränderung des adeligen Bildungsideals und absolutistischer Tendenzen für deren Entstehung.

Anschließend wurde näher auf die Routen der Reisenden eingegangen, wobei festgestellt wurde, dass diese auch von der geographischen Herkunft der Reisenden abhingen, Italien und Frankreich aber klar zu den favorisierten Zielen zählten, während das Römisch-Deutsche Reich nicht zuletzt aufgrund der Verwüstungen durch den Dreißigjährigen Krieg kaum besucht wurde.

Der nächste Abschnitt widmete sich den vielfältigen Funktionen der Kavalierstour für die Reisenden. Es wurde die Distinktionsfunktion ebenso erwähnt wie der Kontakt zur höfischen Welt und das Knüpfen von Beziehungsnetzwerken. Auch der Initiationscharakter der Tour und die Rolle für den Statuserwerb wurden besprochen.

Abschließend wurde näher auf die Rolle der Tutoren eingegangen, nicht zuletzt, weil sie es in den meisten Fällen waren, die die Reiseberichte verfassten. Es wurde auf ihre unterschiedlichen Bezeichnungen und ständische Herkunft hingewiesen, auf ihren Bildungshintergrund, ihre schwierige Rolle als Erzieher der Kavaliere und auf die Chancen in Hinblick auf Bildung und Karriere, die für sie mit der Kavalierstour verbunden waren.

Das nächste Kapitel widmete sich frühneuzeitlicher Reiseliteratur und der Methodisierung des Reisens, wobei auf den Zusammenhang verschiedener historischer Entwicklungen zu Beginn der Neuzeit und Veränderungen in Bezug auf die Reiseliteratur sowie die Entstehung der Methodisierung des Reisens hingewiesen wurde. Insbesondere wurde die Verbindung dieser Entwicklungen mit einem Mentalitätswandel, der zu einer geänderten Wahrnehmung von Fremdheit führte, herausgestellt.

Auf den Aspekt der "Fremde“ in der Frühen Neuzeit wurde im letzten Kapitel des Theorieteils noch näher eingegangen. Hier wurde vor allem betont, dass Fremdheitswahrnehmung sehr schwer zu rekonstruieren ist, sich lediglich Hinweise darauf feststellen lassen.

Der Analyseteil unterteilte sich in die Quellenkritik und eine Inhaltsanalyse nach dem Modell von Philipp Mayering. Die Analyse kam zum Schluss, dass vor allem folgende kulturelle Themen im Reisebericht behandelt wurden: Bauwerke, Architektur und bildende Kunst, Anekdoten, Erzählungen, Sagen und Legenden und "Geschichtliches" sowie Angaben zur "staatlichen“ Organisation der bereisten Gebiete, bei denen es aber kaum Hinweise auf Fremdheitserfahrungen gab.

Außerdem konnte festgestellt werden, dass Themen wie Sprache, Kleidung und Tracht sowie Nahrung und Tischsitten zwar wenig Platz im Bericht einnehmen, in den seltenen Fällen ihrer Erwähnung aber sehr häufig Hinweise auf Fremdheitserfahrungen auftauchen. Somit kann Treues These in Bezug auf die zentrale Rolle von kulinarischen Belangen für die Fremdheitswahrnehmung bestätigt werden. 
Religiöse und konfessionelle Aspekte wurden verhältnismäßig wenig behandelt und es fanden sich dabei auch nur bei weniger als einem Drittel der Erwähnungen Hinweise auf Fremdheitserfahrungen, womit die Gültigkeit der Thesen zu Religion und Konfession für den untersuchten Reisebericht nicht bestätigt werden konnten.

Ridder-Symoens These bezüglich der Rolle der frühneuzeitlichen Reiseberichte für die Entstehung nationaler Stereotype konnte im Hinblick auf den Reisebericht Keyßlers nur bedingt bestätigt werden. Es finden sich im Text zwar verallgemeinernde Aussagen über den Charakter von Gruppen, fraglich ist jedoch, ob diese aufgrund der geringen Häufigkeit im großen Umfang des Reiseberichts von so großem Gewicht waren. Es kann somit festgehalten werden: Der untersuchte Reisebericht mag seinen Beitrag zur Verbreitung von Stereotypen und Vorurteilen geleistet haben, aber nur in geringem Umfang.

Treues These wiederum, dass das Geschlechterverhältnis und die Lebenssituation von Frauen genau beobachtet wurden, konnte durchaus bestätigt werden - es finden sich im Text zwar nicht viele Stellen, an denen diese Themen angesprochen werden, wenn, dann sind aber meistens Hinweise auf Fremdheitserfahrungen zu finden. Es wurde vermutlich also sehr genau beobachtet und diesbezügliche Abweichungen genau registriert und festgehalten.

\section{Literatur- und Quellen}

Allport, Gordon W., Die Natur des Vorurteils, in: Klaus Alheim (Hrsg.), Die Gewalt des Vorurteils. Eine Textsammlung (Politik und Bildung 44), Schwalbach, Ts. 2007, S. 40-59.

Asch, Ronald G., Europäischer Adel in der Frühen Neuzeit. Eine Einführung, Köln-Weimar-Wien 2008.

Brenner, Peter J., Die Erfahrung der Fremde. Zur Entwicklung einer Wahrnehmungsform in der Geschichte des Reiseberichts, in: Peter J. Brenner (Hrsg.), Der Reisebericht in der deutschen Literatur, Frankfurt a. M. 1989, S. 14-49.

Brenner, Peter J., Einleitung, in: Peter J. Brenner (Hrsg.), Der Reisebericht in der deutschen Literatur, Frankfurt a. M. 1989, S. 7-13.

Hahn, Hans Hennig/Hahn, Eva, Nationale Stereotypen. Plädoyer für eine historische Stereotypenforschung, in: Hans Henning Hahn/Stephan Scholz (Hrsg.), Stereotype, Identität und Geschichte. Die Funktion von Stereotypen in gesellschaftlichen Diskursen (Mitteleuropa - Osteuropa. Oldenburger Beiträge zur Kultur und Geschichte Ostmitteleuropas 5), Frankfurt a. M. 2002, S. 17-56.

Keller, Katrin, Zwischen Zeremoniell und "desbauche”. Die adlige Kavalierstour um 1700, in: Wolfgang Schmale/Reinhard Stauber (Hrsg.), Menschen und Grenzen in der Frühen Neuzeit (Innovationen. Bibliothek zur Neueren und Neuesten Geschichte 2), Berlin 1998. 
Keyßler, Johann Georg, Neueste Reisen durch Deutschland, Böhmen, Ungarn, die Schweiz, Italien und Lothringen [...], Hannover 1751, S. XIX-LXXVII, [https:// books.google.at/books/about/Johann_Georg_Keysslers_Neueste_Reisen_ du.html?id=W9AWAAAAQAAJ\&redir_esc=y], eingesehen 24.8.2017.

Lipiansky, Edmund Marc, Die Komplexität der Vorstellung vom Anderen, in: Hans Nicklas/Burkhard Müller/Hagen Kordes (Hrsg.), Interkulturell denken und handeln. Theoretische Grundlagen und gesellschaftliche Praxis, Frankfurt a. M.-New York 2006, S. 117-120.

Maurer, Michael, ,Nationalcharakter' in der frühen Neuzeit. Ein mentalitätsgeschichtlicher Versuch, in: Helmut Kuzmics/Reinhard Blomert/Annette Treibel (Hrsg.), Transformationen des Wir-Gefühls. Studien zum nationalen Habitus, Frankfurt a. M. 1993, S. $45-81$.

Mayering, Philipp, Qualitative Inhaltsanalyse, Weinheim-Basel 2012.

Neuber, Wolfgang, Zur Gattungspoetik des Reiseberichts. Skizzen einer historischen Grundlegung im Horizont von Rhetorik und Topik, in: Peter J. Brenner (Hrsg.), Der Reisebericht in der deutschen Literatur, Frankfurt a. M. 1989, S. 50-67.

Paravicini, Werner, Von der Heidenfahrt zur Kavalierstour. Über Motive und Formen adligen Reisens im späten Mittelalter, in: Ulf-Christian Ewert/Andreas Ranft/Stepan Selzer (Hrsg.), Noblesse. Studien zum Adeligen Leben im Spätmittelalterlichen Europa, Ostfildern 2012, S. 131-170.

Paravicini, Werner, Vom Erkenntniswert der Adelsreise: Einleitung, in: Rainer Babel/Werner Paravicini (Hrsg.), Grand Tour. Adliges Reisen und Europäische Kultur vom 14. bis zum 18. Jahrhundert. Akten der internationalen Kolloquien in der Villa Vigoni 1999 und im Deutschen Historischen Institut Paris 2000 (Beihefte der Francia 60), Ostfildern 2005, S. 11-20.

Ridder-Symoens, Hilde de, Die Kavalierstour im 16. und 17. Jahrhundert, in: Peter J. Brenner (Hrsg.), Der Reisebericht. Die Entwicklung einer Gattung in der deutschen Literatur, Frankfurt a. M. 1989, S. 197-223.

Ridder-Symoens, Hilde de, Mobilität, in: Walter Rüegg (Hrsg.), Geschichte der Universität in Europa II: Von der Reformation bis zur Französischen Revolution 1500-1800, München 1996, S. 335-359.

Schulze, Winfried, Die Entstehung des nationalen Vorurteils. Zur Kultur der Wahrnehmung fremder Nationen in der europäischen Frühen Neuzeit, in: Wolfgang Schmale/ Reinhard Stauber (Hrsg.), Menschen und Grenzen in der Frühen Neuzeit (Innovationen. Bibliothek zur Neueren und Neuesten Geschichte 2), Berlin 1998, S. 23-49.

Schütze, Gottfried, Vorrede, in: Johann Georg Keyßler, Neueste Reisen durch Deutschland, Böhmen, Ungarn, die Schweiz, Italien und Lothringen [...], Hannover 1751, S. I-XVI, [https://books.google.at/books/about/Johann_Georg_Keysslers_Neueste_Reisen_ du.html?id=W9AWAAAAQAAJ\&redir_esc=y], eingesehen 24.8.2017. 
Siebers, Winfried, Johann Georg Keyßler und die Reisebeschreibung der Frühaufklärung, Würzburg 2009.

Stagl, Justin, Eine Geschichte der Neugier. Die Kunst des Reisens 1550-1800, WienKöln-Weimar 2002.

Stannek, Antje, Telemachs Brüder. Die höfische Bildungsreise des 17. Jahrhunderts (Geschichte und Geschlechter 33), Frankfurt a. M. 2001.

Treue, Wolfgang, Abenteuer und Anerkennung. Reisende und Gereiste im Spätmittelalter und Früher Neuzeit, Paderborn 2014.

David Winkler-Ebner ist Master-Student der Geschichte an der Universität Innsbruck, arbeitet im Literaturhaus am Inn in der Programmgestaltung und als freier Mitarbeiter für Ö1. David.Winkler-Ebner@uibk.ac.at

\section{Zitation dieses Beitrages}

David Winkler-Ebner, Kavalierstour: Kulturkontakte und Fremdheitserfahrungen, in: historia.scribere 10 (2018), S. 291-318, [http://historia.scribere.at], eingesehen 19.6.2018 (=aktuelles Datum).

(C) Creative Commons Licences 3.0 Österreich unter Wahrung der Urheberrechte der Autorlnnen. 